Discrete Comput Geom 33:645-668 (2005)

DOI: $10.1007 / \mathrm{s} 00454-005-1164-4$

\title{
The Topological Representation of Oriented Matroids
}

\author{
Jürgen Bokowski, ${ }^{1}$ Simon King, ${ }^{1}$ Susanne Mock, ${ }^{1}$ and Ileana Streinu ${ }^{2}$ \\ ${ }^{1}$ Department of Mathematics, Technische Universität Darmstadt, \\ Schloßgartenstraße 7, 654289 Darmstadt, Germany \\ \{bokowski,king,mock\}@mathematik.tu-darmstadt.de \\ ${ }^{2}$ Department of Computer Science, Smith College, \\ Northampton, MA 01063, USA \\ streinu@cs.smith.edu
}

\begin{abstract}
We present a new proof of the Topological Representation Theorem for oriented matroids in the general rank case. Our proof is based on an earlier rank 3 version. It uses hyperline sequences and the generalized Schönflies theorem. As an application, we show that one can read off oriented matroids from arrangements of embedded spheres of codimension one, even if wild spheres are involved.
\end{abstract}

\section{Introduction}

When studying vector configurations or central hyperplane configurations, point sets on a sphere or great hypersphere arrangements, vector spaces or their duals, points on grassmannians, polytopes and their corresponding cellular decompositions in projective space, etc., an abstraction of an important equivalence class of matrices often plays a central role: an oriented matroid. The theory of oriented matroids (see [1]) provides us with a multitude of definitions for an oriented matroid that can be viewed as reflecting the variety of objects that a matrix can represent.

These definitions via circuit or cocircuit axioms [1, p. 103], sphere systems [1, p. 227], Graßmann-Plücker relations (chirotope axioms) [1, pp. 126, 138], [12], hull systems [15], to mention just a few of them, differ a lot with respect to their motivational aspects, their algorithmic efficiency or their relation to the actual application. Each definition in general provides an additional insight for the motivating problem. In the research monograph on oriented matroids [1], three chapters are devoted to axiomatics concerning oriented matroids and to the Topological Representation Theorem for oriented matroids (TRT, for brevity). 
In this paper we treat three aspects of oriented matroid theory. First, we give a new axiomatic related to the concept of hyperline sequences and show that it is equivalent to the axiomatic of chirotopes. Hyperline sequences provide a rather efficient representation of an oriented matroid. Secondly, we present a new proof of the TRT, based on hyperline sequences. Thirdly, we show that one can read off an oriented matroid from arrangements of embedded spheres of codimension one, even if wild spheres are involved. This was proven by Hochstättler [13] by a much longer argument.

The TRT due to Lawrence is central in the theory of oriented matroids. It shows the equivalence of oriented matroids defined via sphere system axioms with oriented matroids defined via covector axioms. This remarkable result asserts that each oriented matroid has a topological representation as an oriented pseudosphere arrangement, even a piecewise-linear one, see [6]. Recently, a topological representation of matroids (rather than oriented matroids) was found in [24].

Other authors [1], [13] have later simplified or complemented the original proof of the TRT, but all use fundamentally the same approach: the face lattice (tope) formalism for oriented matroids and a shelling order to carry through the construction. Finding a reasonably direct proof in the planar case (rank 3 ) has been posed as an open problem in the research monograph [1] (Exercise 6.3). In [3] such a proof was given, based on hyperline sequences that are particularly natural in rank 3 . In this article we generalize this proof to the arbitrary rank case. The proof is inductive, direct and uses only one advanced result from topology, the generalized Schönflies theorem.

A hyperline sequence represents a rank 2 contraction of an oriented matroid. To motivate the notion of hyperline sequences geometrically, we consider a vector arrangement $V=\left\{v_{1}, \ldots, v_{n}\right\} \subset \mathbb{R}^{r}$ of unit vectors that span $\mathbb{R}^{r}$, with $r \geq 2$. Let $B \subset \mathbb{R}^{r}$ be an oriented subspace of codimension two spanned by $V \cap B$. We obtain a vector arrangement $V_{B}=V \cap B$ in $\mathbb{R}^{r-2}$. The orthogonal complement $C$ of $B$ is a plane, that is oriented according to the orientation of $B$ and of $\mathbb{R}^{r}$. The orthogonal projection of $V \backslash V_{B}$ to $C$ is an ordered set of non-zero vectors which give rise to an ordered set $L_{B}$ of oriented lines in $\mathbb{R}^{2}$. When we move along a circle in $C$ around the origin according to the orientation of $C$, we meet the elements of $V_{C}$ in a circular sequence $Z_{B}$, where any element of $V_{C}$ is met twice (in positive and negative orientation). By an inductive definition (compare Section 2), the hyperline sequence of rank $r$ associated to $B$ is the pair $\left(Y_{B} \mid Z_{B}\right)$, where $Y_{B}$ is the oriented matroid of rank $r-2$ associated to $V_{B}$. The oriented matroid of rank $r$ associated to $V$ is the set of all hyperline sequences that can be read off from $V$.

We chose an inductive definition since it naturally fits into the framework of our proof of the TRT. In fact, it is not necessary to keep complete information on the rank $r-2$ oriented matroids $Y_{B}$. If one chooses a single positively oriented base $\sigma_{B}$ for any hyperline sequence $\left(Y_{B} \mid Z_{B}\right)$ of an oriented matroid $X$ then $X$ is determined by the set of pairs $\left(\sigma_{B} \mid Z_{B}\right)$-see Section 2. Hence a direct definition of oriented matroids in terms of hyperline sequences is possible, which might be preferable for algorithmic problems, e.g., the extension of oriented matroids [18].

The paper is organized as follows. We define hyperline sequences in Section 2. We recall the chirotope concept in Section 3 and we show its equivalence to oriented matroids given by hyperline sequences in Theorem 1 . We introduce the topological representation of oriented matroids via arrangements of oriented pseudospheres in Section 4. We use the generalized Schönflies theorem to show the cellular structure of an arrangement of 
oriented pseudospheres. In Theorem 3 we replace our two axioms for an arrangement of oriented pseudospheres with a single one. Section 5 deals with the easier part of the TRT: we obtain chirotopes and hyperline sequences from the topological representation. We complete the proof of the one-to-one correspondence of oriented matroids with their topological representation by induction. Section 6 is devoted to the base cases and Section 7 contains the essential part. We finally discuss in Section 8 the wild arrangement case as an easy supplement of our approach.

The proof is based on the ideas that have been worked out in [3]. The topological experience of the second author was decisive in arriving at our final version of the proof in the general rank case. The last section and many improvements of the proof compared with the rank 3 version are due to him. For instance, the uniform and non-uniform cases no longer form separate parts within the proof.

\section{Hyperline Sequences}

The aim of this section is to express the notion of oriented matroids in terms of hyperline sequences. The geometric motivation of our definition comes from vector arrangements in Euclidean space as explained in the Introduction.

Let $(E,<)$ be a finite totally ordered set. Let $\bar{E}=\{\bar{e} \mid e \in E\}$ be a copy of $E$. The set $\mathbf{E}$ of signed indices is defined as the disjoint union of $E$ and $\bar{E}$. By extending the map $e \mapsto \bar{e}$ to $\bar{e} \mapsto \overline{\bar{e}}=e$ for $e \in E$, we get an involution on $\mathbf{E}$. We define $e^{*}=\bar{e}^{*}=e$. For $X \subset \mathbf{E}$, define $\bar{X}=\{\bar{x} \mid x \in X\}$ and $X^{*}=\left\{x^{*} \mid x \in X\right\}$.

An oriented $d$-simplex in $E$ is a $(d+1)$-tuple $\sigma=\left[x_{1}, \ldots, x_{d+1}\right]$ of elements of $\mathbf{E}$ such that $x_{1}^{*}, \ldots, x_{d+1}^{*}$ are pairwise distinct. Let an equivalence relation $\sim$ on oriented $d$ simplices in $E$ be generated by $\left[x_{1}, \ldots, x_{d+1}\right] \sim\left[x_{1}, \ldots, x_{i-1}, \overline{x_{i+1}}, x_{i}, x_{i+2}, \ldots, x_{d+1}\right]$, for $i=1, \ldots, d$. As usual, any oriented $d$-simplex is equivalent to one of the form $\left[e_{1}, \ldots, e_{d+1}\right]$ or $\left[e_{1}, \ldots, e_{d}, \overline{e_{d+1}}\right]$, with elements $e_{1}<e_{2}<\ldots<e_{d+1}$ of $E$. Define $-\left[x_{1}, \ldots, x_{d+1}\right]=\left[x_{1}, \ldots, x_{d}, \overline{x_{d+1}}\right]$. In the following inductive definition of hyperline sequences and oriented matroids, we denote with $C_{m}=(\{0,1, \ldots, m-1\},+)$ the cyclic group of order $m$.

Definition 1 (Rank 1). An oriented matroid $X$ over $E(X) \subset E$ of rank 1 is a nonempty subset $X \subset E(X) \cup \overline{E(X)}$ such that $|X|=\left|X^{*}\right|$ and $X^{*}=E(X)$.

The oriented simplex $[x]$ is by definition a positively oriented base of $X$ for any $x \in X$. We define $-X=\bar{X}$.

Definition 2 (Rank 2). Let $k \in \mathbb{N}, k \geq 2$. A hyperline sequence $X$ of rank 2 over $E(X) \subset E$ is a map from $C_{2 k}$ to oriented matroids of rank $1, a \mapsto X^{a}$, such that $X^{a+k}=-X^{a}$ for all $a \in C_{2 k}$, and $E(X) \cup \overline{E(X)}$ is a disjoint union of $X^{0}, \ldots, X^{2 k-1}$.

An oriented matroid of rank 2 is by definition a hyperline sequence of rank 2 . We refer to $X^{0}, \ldots, X^{2 k-1}$ as the atoms of $X$ and to $2 k$ as the period length of $X$. We say that $e \in E(X)$ is incident to an atom $X^{a}$ of $X$ if $e \in\left(X^{a}\right)^{*}$. Let $x_{1}, x_{2} \in E(X) \cup \overline{E(X)}$ such that $x_{1}^{*}$ and $x_{2}^{*}$ are not incident to a single atom of $X$, and $X$ induces the cyclic order $\left(x_{1}, x_{2}, \overline{x_{1}}, \overline{x_{2}}\right)$. Then the oriented simplex $\left[x_{1}, x_{2}\right]$ is by definition a positively oriented 
base of $X$. We define the hyperline sequence $-X$ over $E(-X)=E(X)$ of rank 2 as the map $a \mapsto(-X)^{a}=X^{-a}$ for $a \in C_{2 k}$.

A hyperline sequence $X$ of rank 2 is determined by the sequence $\left(X^{0}, \ldots, X^{2 k-1}\right)$ of atoms. We define that two hyperline sequences $X_{1}$ and $X_{2}$ of rank 2 are equal, $X_{1}=X_{2}$, if $E\left(X_{1}\right)=E\left(X_{2}\right)$, the number $2 k$ of atoms coincides, and $X_{1}$ is obtained from $X_{2}$ by a shift, i.e., there is an $s \in C_{2 k}$ with $X_{1}^{a+s}=X_{2}^{a}$ for all $a \in C_{2 k}$.

We prepare the axioms for oriented matroids of rank $r>2$ with the following definitions. A hyperline sequence $X$ of rank $r$ is a pair $(Y \mid Z)$, where $Y$ is an oriented matroid of rank $r-2$ and $Z$ is a hyperline sequence of rank 2 . If $X$ is a set of hyperline sequences of rank $r$, a positively oriented base of $X$ in $(Y \mid Z) \in X$ is an oriented simplex $\left[x_{1}, \ldots, x_{r}\right]$ in $E(X)$, where $\left[x_{1}, \ldots x_{r-2}\right]$ is a positively oriented base of $Y$ and $\left[x_{r-1}, x_{r}\right]$ is a positively oriented base of $Z$. Then $-\left[x_{1}, \ldots, x_{r}\right]$ is a negatively oriented base of $X$. We define $-X=\{(Y \mid-Z) \mid(Y \mid Z) \in X\}$. An atom of $X$ in a hyperline sequence $(Y \mid Z) \in X$ is the pair $\left(Y \mid Z^{a}\right)$, where $Z^{a}$ is an atom of $Z$.

Definition 3 (Rank $r>2$ ). A set $X$ of hyperline sequences of rank $r$ is an oriented matroid of rank $r>2$ over $E(X) \subset E$ if it satisfies the following axioms:

(H1) $E(X)$ is a disjoint union of $E(Y)$ and $E(Z)$, for all $(Y \mid Z) \in X$.

(H2) Let $\left(Y_{1} \mid Z_{1}\right),\left(Y_{2} \mid Z_{2}\right) \in X$ and let $\left[x_{1}, \ldots x_{r-2}\right]$ be a positively oriented base of $Y_{1}$. If $\left\{x_{1}^{*}, \ldots, x_{r-2}^{*}\right\} \subset E\left(Y_{2}\right)$ then $\left(Y_{1} \mid Z_{1}\right)=\left(Y_{2} \mid Z_{2}\right)$ or $\left(Y_{1} \mid Z_{1}\right)=$ $\left(-Y_{2} \mid-Z_{2}\right)$.

(H3) For all positively oriented bases $\left[x_{1}, \ldots, x_{r}\right]$ and $\left[y_{1}, \ldots, y_{r}\right]$ of $X$, there is some $j \in\{1, \ldots, r\}$ such that $\left[x_{1}, \ldots, x_{r-1}, y_{j}\right]$ is a positively or negatively oriented base of $X$.

(H4) For any positively oriented base $\left[x_{1}, \ldots, x_{r}\right]$ of $X,\left[x_{1}, \ldots, x_{r-3}, \overline{x_{r-1}}, x_{r-2}, x_{r}\right]$ is a positively oriented base of $X$.

We connect these axioms to the geometric motivation exposed in the Introduction. Let $V=\left\{v_{1}, \ldots, v_{n}\right\} \subset \mathbb{R}^{r}, B \subset \mathbb{R}^{r}, Y_{B}, L_{B}$ and $Z_{B}$ be as in the Introduction. To any $v_{k} \in V \backslash Y_{B}$ we get an oriented line $l_{k} \in L_{B}$. We move along a circle in the oriented plane and store the letter $k$ in the circular sequence $Z_{B}$ when $l_{k}$ is met in positive orientation, and $\bar{k}$ if $l_{k}$ is met in negative orientation. Obviously $k$ and $\bar{k}$ appear on opposite places of the circular sequence. Hence $Z_{B}$ is a hyperline sequence of rank 2 . By induction and abuse of notation, the vector arrangement $Y_{B}$ "is" an oriented matroid $Y_{B}$, and $\left(Y_{B} \mid Z_{B}\right)$ is a hyperline sequence of rank $r$. Axiom (H1) means that $V$ is a disjoint union of $V \cap B$ and $V \backslash B$. Axiom (H2) corresponds to the fact that $B$ is determined by any oriented base of $V_{B}$. Axiom (H3) is the Steinitz-McLane exchange lemma, stating that one can replace any vector in a base by some vector of any other base. Axiom (H4) ensures that the definition of oriented bases is compatible with the equivalence relation on oriented simplices; this is part of Theorem 1 below. Axiom (H4) is related to the "consistent abstract sign of determinant" in [3]. It means that if $r$ vectors span an $(r-1)$-simplex, then any subset of $r-2$ vectors spans a hyperline, and the orientation of the $(r-1)$ simplex does not depend on the hyperline on which we consider the $r$ points. A hyperline sequence stores information on a rank 2 contraction of an oriented matroid.

Whereas the inductive definition of oriented matroids in terms of hyperline sequences fits well to the structure of our proof of the TRT, a direct definition might be profitable for 
algorithmic use. We sketch such a direct approach in the rest of this section. If $(Y \mid Z)$ is a hyperline sequence in an oriented matroid $X$ of rank $r$ over $E$, then we can describe the oriented matroid $Y$ of rank $r-2$ by the set of its positively oriented bases, according to Theorem 1 in the next section. A positively oriented base $\sigma$ of $Y$ is equivalent to an oriented simplex of the form $\pm\left[b_{1}, \ldots, b_{r-2}\right]$ with $b_{1}<b_{2}<\cdots<b_{r-2}$ and $B_{\sigma}:=\left\{b_{1}, \ldots, b_{r-2}\right\} \subset E$. Hence, $(Y \mid Z)$ uniquely corresponds to a set of triples $\left\{\left(B_{\sigma} ; E \backslash B_{\sigma} \mid Z_{\sigma}\right)\right\}_{\sigma}$, where $\sigma$ runs over all equivalence classes of positively oriented bases of $Y$, and $Z_{\sigma}=Z$ for $\sigma \sim\left[b_{1}, \ldots, b_{r-2}\right]$ and $Z_{\sigma}=-Z$ for $\sigma \sim-\left[b_{1}, \ldots, b_{r-2}\right]$. Note that $\left\{\left(B_{\sigma} ; E \backslash B_{\sigma} \mid Z_{\sigma}\right)\right\}_{\sigma}$ just contains a single element if the oriented matroid $X$ is uniform. We proceed in that way for all hyperline sequences in $X$ and obtain a set of triples $\{(B ; J \mid Z)\}$, with disjoint subsets $B, J \subset E$ and a hyperline sequence $Z$ of rank 2 over $E \backslash(B \cup J)$ of period length at least 4.

Conversely, let $\tilde{X}=\{(B ; J \mid Z)\}$ be a set of triples, where $B$ and $J$ are disjoint subsets of $E,|B|=r-2$, and $Z$ is a hyperline sequence of rank 2 over $E \backslash(B \cup J)$ of period length at least 4 . Let $L=(B ; J \mid Z) \in \tilde{X}$ and $B=\left\{b_{1}, \ldots, b_{r-2}\right\}$ with $b_{1}<\ldots<b_{r-2}$. For $i, j \in E(Z)$ that are not incident to the same atom of $Z$, we define $L^{i, j}=\left[b_{1}, \ldots, b_{r-2}, i, j\right]$ if $Z$ induces the cyclic order $(i, j, \bar{l}, \bar{J})$, and $L^{i, j}=$ $-\left[b_{1}, \ldots, b_{r-2}, i, j\right]$ otherwise. We call an oriented simplex equivalent to one of the form $L^{i, j}$ for $L \in \tilde{X}$ an oriented base of $\tilde{X}$. We claim that $\tilde{X}$ corresponds to an oriented matroid of rank $r>1$ if and only if it satisfies the following axioms:

(H1') For any $i \in E$ there is some $(B ; J \mid Z) \in \tilde{X}$ with $i \in B$.

$\left(\mathrm{H} 2^{\prime}\right)$ Let $\left(B_{1} ; J_{1} \mid Z_{1}\right),\left(B_{2} ; J_{2} \mid Z_{2}\right) \in \tilde{X}$. If $B_{1} \subset B_{2} \cup J_{2}$ then $Z_{1}= \pm Z_{2}$. If $B_{1}=B_{2}$ then $Z_{1}=Z_{2}$.

$\left(\mathrm{H}^{\prime}\right)$ For any oriented bases $\left[x_{1}, \ldots, x_{r}\right]$ and $\left[y_{1}, \ldots, y_{r}\right]$ of $\tilde{X}$, there is some $j \in$ $\{1, \ldots, r\}$ such that $\left[x_{1}, \ldots, x_{r-1}, y_{j}\right]$ is an oriented base of $\tilde{X}$.

(H4') For any $L_{1}=\left(B_{1} ; J_{1} \mid Z_{1}\right) \in \tilde{X}$, any $i, j \in E \backslash\left(B_{1} \cup J_{1}\right)$ that are not incident to the same atom of $Z_{1}$, and any $k \in B_{1}$, there is some $L_{2}=$ $\left(\{i\} \cup B_{1} \backslash\{k\} ; J_{2} \mid Z_{2}\right) \in \tilde{X}$ such that $j, k \in E\left(Z_{2}\right)$, are not incident to the same atom of $Z_{2}$, and $L_{1}^{i, j} \sim L_{2}^{j, k}$.

To prove that Axioms $\left(\mathrm{H}^{\prime}\right)-\left(\mathrm{H} 4^{\prime}\right)$ correspond to Axioms $(\mathrm{H} 1)-(\mathrm{H} 4)$, respectively, is left to the reader. We remark that storing an oriented matroid in that way is still more economical than just storing all positively oriented bases, even in the non-uniform case. Moreover, it will be clear from the proof of Theorem 1 in the next section that the cyclic order of a single hyperline sequence already captures many instances of the GrassmannPlücker relations. Thus when enumerating oriented matroids, it seems easier to produce a set of hyperline sequences and verify Axioms $\left(\mathrm{H1}^{\prime}\right)-\left(\mathrm{H} 4^{\prime}\right)$ than to produce a list of oriented simplices and verify, say, the chirotope axioms.

\section{Chirotopes}

We recall in this section the chirotope axioms for oriented matroids (see pp. 126 and 138 of [1] and see [12]). Let $(E,<)$ be as in the preceding section. We denote by $\Delta_{d}(E)$ the set of all oriented $d$-simplices in $E$. 
Definition 4. A chirotope $\chi$ of rank $r$ over $E$ is a map $\Delta_{r-1}(E) \rightarrow\{-1,0,+1\}$, such that the following holds:

(C1) For any $e_{1} \in E$, there are $e_{2}, e_{3}, \ldots, e_{r} \in E$ with $\chi\left(\left[e_{1}, \ldots, e_{r}\right]\right) \neq 0$.

(C2) For any $\sigma \in \Delta_{r-1}(E), \chi(-\sigma)=-\chi(\sigma)$ holds, and if $\sigma \sim \tau$ then $\chi(\sigma)=$ $\chi(\tau)$.

(C3) If $\chi\left(\left[x_{1}, \ldots, x_{r}\right]\right) \neq 0$ and $\chi\left(\left[y_{1}, \ldots, y_{r}\right]\right) \neq 0$ then there is some $i \in$ $\{1, \ldots, r\}$ with $\chi\left(\left[x_{1}, \ldots, x_{r-1}, y_{i}\right]\right) \neq 0$.

(C4) If $x_{1}, x_{2}, \ldots, x_{r}, y_{1}, y_{2} \in \mathbf{E}$ so that

$$
\begin{aligned}
& \chi\left(\left[x_{1}, \ldots, x_{r-2}, y_{1}, x_{r}\right]\right) \cdot \chi\left(\left[x_{1}, \ldots, x_{r-1}, y_{2}\right]\right) \geq 0 \text { and } \\
& \chi\left(\left[x_{1}, \ldots, x_{r-2}, y_{2}, x_{r}\right]\right) \cdot \chi\left(\left[x_{1}, \ldots, x_{r-1}, \overline{y_{1}}\right]\right) \geq 0,
\end{aligned}
$$

then $\chi\left(\left[x_{1}, \ldots, x_{r}\right]\right) \cdot \chi\left(\left[x_{1}, \ldots, x_{r-2}, y_{1}, y_{2}\right]\right) \geq 0$.

A simplex $\sigma \in \Delta_{r-1}(E)$ is a positively oriented base of $\chi$ if $\chi(\sigma)=+1$. By Axiom (C2), $\chi$ is completely described by the set of its positively oriented bases. It is well known that the positively oriented bases of an arrangement of unit vectors $V \subset \mathbb{R}^{r}$ form the positively oriented bases of a chirotope of rank $r$. In the setting of vector arrangements, Axiom (C3) is the Steinitz-McLane exchange lemma, as Axiom (H3) above. Axiom (C4) says that the signs of oriented bases of $V$ do not contradict the three summand Graßmann-Plücker relations.

We recall the notions of deletion and contraction, which are essential in our inductive proof of TRT. Let $\chi$ be a chirotope of rank $r$ over $E$, and let $R \subset E$. Then the deletion of $R$ in $\chi$ is the restriction $\chi \backslash R: \Delta_{r-1}(E \backslash R) \rightarrow\{-1,0,+1\}$ of $\chi$ to $\Delta_{r-1}(E \backslash R)$. Unless any positively oriented base of $\chi$ contains an element of $R, \chi \backslash R$ is a chirotope. It is well known and not difficult to prove that if $|E|>r$ then there is an $i \in E$ so that $\chi \backslash\{i\}$ is a chirotope of rank $r$ over $E \backslash\{i\}$.

Let $R=\left\{e_{1}, \ldots, e_{k}\right\} \subset E$, with $e_{1}<\ldots<e_{k}$ and $k<r$. We define $E / R$ as the set of all $e \in E$ for which there exist $e_{k+1}, \ldots, e_{r-1} \in E$ such that $\chi\left(\left[e_{1}, \ldots, e_{r-1}, e\right]\right) \neq 0$. If $E / R \neq \emptyset$ (i.e., $R$ is an independent set) the contraction of $\chi$ on $R$ is the map $\chi / R: \Delta_{r-1-k}(E / R) \rightarrow\{-1,0,+1\}$ by $\chi / R\left(\left[e_{k+1}, \ldots, e_{r}\right]\right)=\chi\left(\left[e_{1}, \ldots, e_{r}\right]\right)$, for all $\left[e_{k+1}, \ldots, e_{r}\right] \in \Delta_{r-1}(E / R)$.

By the following theorem, chirotopes and hyperline sequences yield equivalent notions of oriented matroids. This connects our concept of hyperline sequences with other ways to look at oriented matroids. The cyclic structure of a hyperline sequence captures many instances of the three-term Graßmann-Plücker relations at once. Certainly it is easier to deal with a few cyclic structures than with a multitude of Graßmann-Plücker relations, specifically in algorithmic applications. There is a price to pay for the simplification in the representation of oriented matroids: the proof of the theorem becomes rather long and tedious if it is carried out in detail.

Theorem 1. The set of positively oriented bases of an oriented matroid of rank $r$ over $E$ given by hyperline sequences is the set of positively oriented bases of a chirotope of rank $r$ over $E$, and vice versa.

Proof. 1. Let $X$ be an oriented matroid of rank $r$ over $E$ given by hyperline sequences, and let $\left[x_{1}, \ldots, x_{r}\right]$ be a positively oriented base of $X$. Since the cyclic orders 
$\left(x_{r-1}, x_{r}, \overline{x_{r-1}}, \overline{x_{r}}\right)$ and $\left(\overline{x_{r}}, x_{r-1}, x_{r}, \overline{x_{r-1}}\right)$ are equal up to a shift, $\left[x_{1}, \ldots, x_{r-2}, \overline{x_{r}}, x_{r-1}\right]$ is a positively oriented base of $X$ as well. This together with Axiom (H4) and an induction on the rank implies that all oriented simplices equivalent to $\left[x_{1}, \ldots, x_{r}\right]$ are positively oriented bases of $X$, hence, yields Axiom (C2). Axiom (C1) follows from Axiom (H1), Axiom (C2) and induction on the rank. Axioms (H3) and (C3) are equivalent.

In the next paragraphs we deduce Axiom (C4) from the cyclic order of hyperline sequences. We tacitly use Axiom (C2), that is already proven. Let $x_{1}, x_{2}, \ldots, x_{r}, y_{1}, y_{2} \in$ E satisfy the first two inequalities in Axiom (C4). The third inequality is to be proven. We can assume that $x_{1}, \ldots, x_{r-2}$ defines some hyperline sequence $(Y \mid Z) \in X$ with period length $2 k$, since otherwise Axiom (C4) is trivial. For simplicity, we write $[a, b]$ in place of $\chi\left(\left[x_{1}, \ldots, x_{r-2}, a, b\right]\right)$.

If $\left[y_{1}, x_{r}\right]=\left[x_{r-1}, y_{2}\right]=-1$ then we replace $y_{1}$ with $x_{r}, x_{r}$ with $y_{1}, x_{r-1}$ with $\overline{y_{2}}$ and $y_{2}$ with $\overline{x_{r-1}}$. This changes the signs of the factors in the first inequality, whereas the other inequalities remain unchanged. Similarly, we can assume without loss of generality that both factors of the second inequality and $\left[y_{1}, y_{2}\right]$ are non-negative. Let $a, b, c, d \in C_{2 k}$ so that $x_{r-1} \in Z^{a}, x_{r} \in Z^{b}, y_{1} \in Z^{c}, y_{2} \in Z^{d}$. By shifting the hyperline sequence, we assume that $c=0$. Then we have $b, d-a, b-d, a, d \in\{0,1, \ldots, k\} \subset C_{2 k}$.

If $\left[y_{1}, y_{2}\right]=0$ then the third inequality is satisfied, and Axiom (C4) is proven. If $\left[y_{1}, y_{2}\right]=1$ then $d \in\{1, \ldots, k-1\}$. Assume that $\left[x_{r-1}, x_{r}\right]=-1$, thus $a-b \in$ $\{1, \ldots, k-1\}$. With the natural order on $0,1, \ldots, k$ we find $0 \leq b<a \leq d<k$. This is a contradiction to $b-d \in\{0,1, \ldots, k\}$. Hence $\left[x_{r-1}, x_{r}\right] \geq 0$, which finishes the proof of Axiom (C4).

2. Conversely, let $\chi$ be a chirotope of rank $r$ over $E$. We wish to construct hyperline sequences, forming an oriented matroid $X$ with the same positively oriented bases. In our proof we do not mention all applications of Axiom (C2) explicitly. If $\chi$ is of rank 1 , then we define $X$ as the set of all $x \in \mathbf{E}$ with $\chi([x])=+1$. Since $\chi([x])=-\chi([\bar{x}])$ and since $X \neq \varnothing$ by Axiom (C1), $X$ is an oriented matroid of rank 1 , and it has the desired positively oriented bases by construction.

It is not difficult to prove that any chirotope $\chi$ of rank 2 is realizable by an arrangement of unit planar vectors. From this, one can read off the cyclic structure of a hyperline sequence. Hence we obtain an oriented matroid of rank 2 with the same positively oriented bases as $\chi$.

There remains the case of rank $r \geq 3$. By Axioms (C1) and (C2), there are $x_{1}, \ldots, x_{r} \in$ $\mathbf{E}$ with $\chi\left(\left[x_{1}, \ldots, x_{r}\right]\right)=1$. It is easy to verify that

$$
C\left(x_{1}, \ldots, x_{r-2}\right):=\left\{\left[y_{r-1}, y_{r}\right] \in \Delta_{1}(E) \mid \chi\left(\left[x_{1}, \ldots, x_{r-2}, y_{r-1}, y_{r}\right]\right)=1\right\}
$$

is the set of positively oriented bases of a rank 2 contraction of $\chi$, thus, of a hyperline sequence $Z\left(x_{1}, \ldots, x_{r-2}\right)$ of rank 2 . We define

$$
\begin{aligned}
B\left(x_{1}, \ldots, x_{r-2}\right):= & \left\{\left[y_{1}, \ldots, y_{r-2}\right] \in \Delta_{r-3}(E) \mid \chi\left(\left[y_{1}, \ldots, y_{r}\right]\right)=1\right. \\
& \text { for some } \left.\left[y_{r-1}, y_{r}\right] \in C\left(x_{1}, \ldots, x_{r-2}\right)\right\} .
\end{aligned}
$$

We claim that $B\left(x_{1}, \ldots, x_{r-2}\right)$ is the set of positively oriented bases of a chirotope of rank $r-2$ over the set of those elements of $E$ that do not occur in $Z\left(x_{1}, \ldots, x_{r-2}\right)$. Axioms $(\mathrm{C} 2)-(\mathrm{C} 4)$ for $B\left(x_{1}, \ldots, x_{r-2}\right)$ are obvious consequences of the corresponding axioms for $\chi$. To prove Axiom (C1) for $B\left(x_{1}, \ldots, x_{r-2}\right)$, let $e \in E \backslash E\left(Z\left(x_{1}, \ldots, x_{r-2}\right)\right)$. 
We chose $\left[x_{r-1}, x_{r}\right] \in C\left(x_{1}, \ldots, x_{r-2}\right)$, hence $\chi\left(\left[x_{1}, \ldots, x_{r}\right]\right)=1$. Since $\chi$ satisfies Axiom (C3), we find some $j \in\{1, \ldots, r\}$ such that $\chi\left(\left[x_{1}, \ldots, x_{j-1}, e, x_{j+1}, \ldots, x_{r}\right]\right) \neq$ 0 . Since $e \notin E\left(Z\left(x_{1}, \ldots, x_{r-2}\right)\right)$, we have $j \leq r-2$. By application of Axiom (C2), we can move $e$ to the first position of the oriented simplex, keeping $x_{r-1}, x_{r}$ at the two last positions, and this yields Axiom (C1) for $B\left(x_{1}, \ldots, x_{r-2}\right)$. By induction, we obtain an oriented matroid $Y\left(x_{1}, \ldots, x_{r-2}\right)$ of rank $r-2$ whose set of positively oriented bases is $B\left(x_{1}, \ldots, x_{r-2}\right)$.

We collect all pairs $\left(Y\left(x_{1}, \ldots, x_{r-2}\right) \mid Z\left(x_{1}, \ldots, x_{r-2}\right)\right)$ to form a set (not a multiset) $X$, where $x_{1}, \ldots, x_{r-2} \in \mathbf{E}$. It has the same positively oriented bases as $\chi$, by construction. We will show that $X$ is an oriented matroid of rank $r$ over $E$. The most difficult to prove is Axiom (H1). Let $Y=Y\left(x_{1}, \ldots, x_{r-2}\right)$ and $Z=Z\left(x_{1}, \ldots, x_{r-2}\right)$. By definition, in an oriented simplex over $E$ any element of $\mathbf{E}$ occurs at most once. This implies $E(Y) \cap E(Z)=\emptyset$. Let $e \in E \backslash E(Z)$. It remains to show that $e \in E(Y)$, hence $E=E(Y) \cup E(Z)$. Let $\left[x_{1}, \ldots, x_{r-2}\right]$ and $\left[x_{r-1}, x_{r}\right]$ be positively oriented bases of $Y$ and $Z$, respectively. Thus $\chi\left(\left[x_{1}, \ldots, x_{r}\right]\right)=1$. By Axioms (C2) and (C3), there is an index $i$ so that $\chi\left(\left[x_{1}, \ldots, x_{i-1}, e, x_{i+1}, \ldots, x_{r}\right]\right) \neq 0$. We have $i<r-1$ since $e \notin E(Z)$. Hence $e \in E(Y)$ by definition of $Y\left(x_{1}, \ldots, x_{r-2}\right)$.

We verify the remaining axioms. Since $\chi\left(\left[x_{1}, \ldots, x_{r}\right]\right)=\chi\left(\left[x_{1}, \ldots, x_{r-3}, \overline{x_{r-2}}, x_{r}\right.\right.$, $\left.x_{r-1}\right]$ ) by Axiom (C2), we have

$$
\begin{aligned}
& Y\left(x_{1}, \ldots, x_{r-3}, \overline{x_{r-2}}\right)=-Y\left(x_{1}, \ldots, x_{r-2}\right), \\
& Z\left(x_{1}, \ldots, x_{r-3}, \overline{x_{r-2}}\right)=-Z\left(x_{1}, \ldots, x_{r-2}\right),
\end{aligned}
$$

and this implies Axiom (H2). Axiom (H3) is Axiom (C3). Finally, Axiom (H4) is a special case of Axiom (C2). Thus, $X$ is an oriented matroid.

This theorem allows one to translate the notions of deletion and contraction to hyperline sequences. If $X$ is an oriented matroid of rank $r$ over $E$ given by hyperline sequences, associated to a chirotope $\chi$, and if $\chi \backslash R$ (resp. $\chi / R$ ) is defined for $R \subset E$, then the deletion $X \backslash R$ of $R$ in $X$ (resp. the contraction $X / R$ of $X$ on $R$ ) is the oriented matroid associated to $\chi \backslash R$ (resp. $\chi / R$ ).

\section{Arrangements of Oriented Pseudospheres}

In the preceding sections we extracted combinatorial data from vector arrangements and turned properties of these data into axioms, yielding hyperline sequences and chirotopes. In this section we generalize vector arrangements in a geometric way. The main aim of this paper is to prove the equivalence of these geometric structures with hyperline sequences.

Let $\mathbb{S}^{r-1} \subset \mathbb{R}^{r}$ be the unit sphere. Any arrangement of non-zero unit vectors in $\mathbb{R}^{r}$ is dual to an arrangement of oriented hyperspheres in $\mathbb{S}^{r-1}$. Since there are oriented matroids that cannot be realized by an arrangement of oriented hyperspheres, one wants to replace hyperspheres by more general objects, namely oriented embedded spheres of codimension one in $\mathbb{S}^{r-1}$.

We formalize this idea. Let $\mathbb{S}^{d}$ denote the $d$-dimensional oriented sphere

$$
\mathbb{S}^{d}=\left\{\left(x_{1}, \ldots, x_{d+1}\right) \in \mathbb{R}^{d+1} \mid x_{1}^{2}+\ldots+x_{d+1}^{2}=1\right\},
$$


and let $B^{d}=\left\{\left(x_{1}, \ldots, x_{d}\right) \in \mathbb{R}^{d} \mid x_{1}^{2}+\ldots+x_{d}^{2} \leq 1\right\}$ denote the closed $d$-dimensional ball. A submanifold $N$ of codimension $m$ in a $d$-dimensional manifold $M$ is tame if any $x \in N$ has an open neighborhood $U(x) \subset M$ such that there is a homeomorphism $\overline{U(x)} \rightarrow B^{d}$ sending $U(x) \cap N$ to $B^{d-m} \subset B^{d}$.

An oriented pseudosphere $S \subset \mathbb{S}^{d}$ is a tame embedded $(d-1)$-dimensional sphere with a choice of an orientation. Any oriented hypersphere is an oriented pseudosphere. The image of an oriented pseudosphere $S$ under a homeomorphism $\varphi: \mathbb{S}^{d} \rightarrow \mathbb{S}^{d}$ obviously is an oriented pseudosphere as well.

We frequently use the generalized Schönflies theorem that was proven by Brown [4]. It assures that $\mathbb{S}^{d} \backslash S$ is a disjoint union of two open balls whose closures are closed balls, if $S$ is a pseudosphere in $\mathbb{S}^{d}$. Another way to state the generalized Schönflies theorem is that for any pseudosphere $S \subset \mathbb{S}^{d}$ there is a homeomorphism $\varphi: \mathbb{S}^{d} \rightarrow \mathbb{S}^{d}$ such that $\varphi(S)=\mathbb{S}^{d-1} \subset \mathbb{S}^{d}$. Similarly, if $B \subset B^{d}$ is a tame embedded ball of codimension one and $\partial B \subset \partial B^{d}$ then there is a homeomorphism $B^{d} \rightarrow B^{d}$ sending $B$ to $B^{d-1} \subset B^{d}$. It is well known that in dimension $d \geq 3$ there are embedded spheres of codimension one whose complement is not formed by two balls, so-called wild spheres.

Remark. PL-embedded spheres of codimension one are tame, and any pseudosphere in $\mathbb{S}^{d}$ can be sent to a PL-embedded sphere by some homeomorphism $\mathbb{S}^{d} \rightarrow \mathbb{S}^{d}$. Hence, one can alternatively define pseudospheres as PL-embedded spheres of codimension one. In fact our proof of the TRT works in the PL-setting as well. Nevertheless, we prefer to deal with tame embeddings rather than PL-embeddings, for the following aesthetic reason. It is still an open question whether any two PL-embedded $(d-1)$-spheres in $\mathbb{S}^{d}$ can be related by a PL-homeomorphism $\mathbb{S}^{d} \rightarrow \mathbb{S}^{d}$. Hence, when translating our construction into the PL setting, we have to assume homeomorphisms that are not necessarily PL.

Let $\psi: \mathbb{S}^{d-1} \rightarrow \mathbb{S}^{d}$ be an embedding with image $S$, inducing the correct orientation on $S$. By another result of Brown [5], the image of $\psi$ is tame if and only if $\psi$ can be extended to an orientation preserving embedding

$$
\tilde{\psi}: \mathbb{S}^{d-1} \times[-1,1] \rightarrow \mathbb{S}^{d} \quad \text { with } \quad \psi(\cdot)=\tilde{\psi}(\cdot, 0) .
$$

We call the connected component of $\mathbb{S}^{d} \backslash S$ containing $\tilde{\psi}\left(\mathbb{S}^{d-1} \times\{1\}\right)$ (resp. $\tilde{\psi}\left(\mathbb{S}^{d-1} \times\right.$ $\{-1\})$ ) the positive side $S^{+}$(resp. negative side $S^{-}$) of $S$.

The following definition of arrangements of oriented pseudospheres is similar to that in [1, p. 227]. Recall $E_{n}=\{1, \ldots, n\}$.

Definition 5. Let $S_{1}, \ldots, S_{n} \subset \mathbb{S}^{d}$ be not necessarily distinct oriented pseudospheres, ordered according to their indices. Assume that the following conditions hold:

(A1) $S_{R}=\mathbb{S}^{d} \cap \bigcap_{i \in R} S_{i}$ is empty or homeomorphic to a sphere, for all $R \subset E_{n}$.

(A2) Let $R \subset E_{n}$ and $i \in E_{n}$ with $S_{R} \not \subset S_{i}$. Then $S_{R} \cap S_{i}$ is a pseudosphere in $S_{R}$, and $S_{R} \cap S_{i}^{+}$and $S_{R} \cap S_{i}^{-}$are both non-empty.

Then the ordered multiset $\left\{S_{1}, \ldots, S_{n}\right\}$ is an arrangement of oriented pseudospheres over $E_{n}$. The arrangement is called of full rank if the intersection of its members is empty. 
We omitted Axiom (A3) from [1, p. 227], since it follows from the other two axioms. In [1], arrangements of full rank are called essential. Obviously if $S_{1}, \ldots, S_{n}$ are oriented hyperspheres then $\left\{S_{1}, \ldots, S_{n}\right\}$ is an arrangement of oriented pseudospheres. At the end of this section we characterize arrangements of oriented pseudospheres by a single axiom.

Definition 6. Two ordered multisets $\left\{S_{1}, \ldots, S_{n}\right\}$ and $\left\{S_{1}^{\prime}, \ldots, S_{n}^{\prime}\right\}$ of oriented pseudospheres in $\mathbb{S}^{d}$ are equivalent if there is an orientation preserving homeomorphism $\mathbb{S}^{d} \rightarrow \mathbb{S}^{d}$ sending $S_{i}^{+}$to $\left(S_{i}^{\prime}\right)^{+}$and $S_{i}^{-}$to $\left(S_{i}^{\prime}\right)^{-}$, simultaneously for all $i \in E_{n}$. We do not allow renumbering of the pseudospheres. We do distinguish the two orientations of $\mathbb{S}^{d}$.

Example 1. We construct non-equivalent arrangements $\mathcal{A}(d,+), \mathcal{A}(d,-)$ of $d+1$ oriented pseudospheres $S_{1}, \ldots, S_{d+1} \subset \mathbb{S}^{d}$ of full rank, corresponding to the two orientations of $\mathbb{S}^{d}$, as follows. For $i=1, \ldots, d+1$, set

$$
S_{i}=\left\{\left(x_{1}, \ldots, x_{d+1}\right) \in \mathbb{S}^{d} \mid x_{i}=0\right\} .
$$

In $\mathcal{A}(d,+)$, define $S_{1}^{+}=\left\{\left(x_{1}, \ldots, x_{d+1}\right) \in \mathbb{S}^{d} \mid x_{1}>0\right\}$. In $\mathcal{A}(d,-)$, define $S_{1}^{+}=$ $\left\{\left(x_{1}, \ldots, x_{d+1}\right) \in \mathbb{S}^{d} \mid x_{1}<0\right\}$. In both $\mathcal{A}(d,+)$ and $\mathcal{A}(d,-)$, define $S_{i}^{+}=\left\{\left(x_{1}, \ldots\right.\right.$, $\left.\left.x_{d+1}\right) \in \mathbb{S}^{d} \mid x_{i}>0\right\}$, for $i=2, \ldots, d+1$.

$\mathcal{A}(d,+)$ and $\mathcal{A}(d,-)$ are of full rank. If $\varphi: \mathbb{S}^{d} \rightarrow \mathbb{S}^{d}$ is any homeomorphism that fixes $S_{2}^{+}, \ldots, S_{d+1}^{+}$setwise and maps $\left\{\left(x_{1}, \ldots, x_{d+1}\right) \in \mathbb{S}^{d} \mid x_{1}>0\right\}$ to $\left\{\left(x_{1}, \ldots, x_{d+1}\right) \in\right.$ $\left.\mathbb{S}^{d} \mid x_{1}<0\right\}$, then $\varphi$ is orientation reversing. Thus, $\mathcal{A}(d,+)$ is not equivalent to $\mathcal{A}(d,-)$.

We re-examine deletion and contraction in the framework of arrangements of oriented pseudospheres. Fix an arrangement $\mathcal{A}=\left\{S_{1}, \ldots, S_{n}\right\}$ of oriented pseudospheres in $\mathbb{S}^{d}$. For any $R \subset E_{n}$, we obtain an arrangement

$$
\mathcal{A} \backslash\left\{S_{r} \mid r \in R\right\}
$$

of oriented pseudospheres over $E_{n} \backslash R$ in $\mathbb{S}^{d}$ (Axioms (A1) and (A2) are easy to verify). We denote this arrangement by $\mathcal{A} \backslash R$ and call it the deletion of $R$ in $\mathcal{A}$. In general, $\mathcal{A} \backslash R$ is not of full rank, even if $\mathcal{A}$ is.

It is intuitively clear from Axiom (A2), that for $R \subset E_{n}$ one gets an arrangement of oriented pseudospheres on $S_{R}$, induced by $\mathcal{A}$. In the following iterative definition of this induced arrangement, the orientation of $S_{R}$ requires some care. Let $r \in E_{n}$, and let $\psi_{r}: \mathbb{S}^{d-1} \rightarrow \mathbb{S}^{d}$ be a tame embedding defining $S_{r}$ with the correct orientation. Denote $S_{i}^{\prime}=\psi_{r}^{-1}\left(S_{i} \cap S_{r}\right)$, for $i \in E_{n}$. We obtain an ordered multiset

$$
\mathcal{A} /\{r\}=\left\{S_{i}^{\prime} \mid i \in E_{n}, S_{r} \not \subset S_{i}\right\}
$$

of oriented pseudospheres in $\mathbb{S}^{d-1}$, where $\left(S_{i}^{\prime}\right)^{+}=\psi_{r}^{-1}\left(S_{i}^{+} \cap S_{r}\right)$. Axioms (A1) and (A2) are easy to verify, thus, $\mathcal{A} /\{r\}$ is an arrangement of oriented pseudospheres over a subset of $E_{n} \backslash\{r\}$.

This construction can be iterated. Let $R \subset E_{n}$ so that $\operatorname{dim} S_{R}=d-|R|$. List the elements of $R$ in ascending order, $r_{1}<r_{2}<\ldots<r_{|R|}$. The contraction of $\mathcal{A}$ in $R$ is then the arrangement of oriented pseudospheres

$$
\mathcal{A} / R=\left(\cdots\left(\left(\mathcal{A} /\left\{r_{1}\right\}\right) /\left\{r_{2}\right\}\right) \cdots /\left\{r_{|R|}\right\}\right) .
$$


Note that $\left(\mathcal{A} /\left\{r_{1}\right\}\right) /\left\{r_{2}\right\}$ and $\left(\mathcal{A} /\left\{r_{2}\right\}\right) /\left\{r_{1}\right\}$ are related by an orientation reversing homeomorphism $\mathbb{S}^{d-2} \rightarrow \mathbb{S}^{d-2}$. It is easy to see that $\mathcal{A} / R$ is of full rank if and only if $\mathcal{A}$ is of full rank.

An arrangement $\mathcal{A}=\left\{S_{1}, \ldots, S_{n}\right\}$ of oriented pseudospheres in $\mathbb{S}^{d}$ yields a cellular decomposition of $\mathbb{S}^{d}$, as follows. For any subset $I \subset E_{n}$, we consider the parts of the intersection of the pseudospheres with label in $I$ that are not contained in pseudospheres with other labels,

$$
\begin{aligned}
\mathcal{C}(I, \mathcal{A}) & =\left\{x \in \mathbb{S}^{d} \mid x \in S_{i} \Longleftrightarrow i \in I, \text { for all } i \in E_{n}\right\} \\
& =\left(\mathbb{S}^{d} \cap \bigcap_{i \in I} S_{i}\right) \backslash\left(\bigcup_{j \in E_{n} \backslash I} S_{j}\right) .
\end{aligned}
$$

By the next theorem, the connected components of $\mathcal{C}(I, \mathcal{A})$ are topological cells if $\mathcal{A}$ is of full rank. Hence, it makes sense to refer to a connected component of $\mathcal{C}(I, \mathcal{A})$ as a cell of $\mathcal{A}$ with label $I$. The $d$-skeleton $\mathcal{A}^{(d)}$ of $\mathcal{A}$ is the union of its cells of dimension $\leq d$. By Axiom (A1), $\bigcap_{i \in R} S_{i}$ is empty or a sphere, for $R \subset E_{n}$. Thus, for any zero-dimensional cell of $\mathcal{A}$ there exists exactly one other cell of $\mathcal{A}$ with the same label, corresponding to the two points in $\mathbb{S}^{0}$. The statement that the cells of an arrangement of oriented pseudospheres of full rank are in fact topological cells is essential for the proof of the TRT in Sections 6 and 7.

Theorem 2. Let $\mathcal{A} \neq \emptyset$ be an arrangement of oriented pseudospheres over $E_{n}$. Any connected component of $\mathcal{C}(\emptyset, \mathcal{A})$ is a d-dimensional cell whose closure is a closed ball. If $\mathcal{A}$ is of full rank, then for any $I \subset E_{n}$, any connected component of $\mathcal{C}(I, \mathcal{A})$ is an open cell whose closure is a closed ball.

Proof. First, we prove that the theorem holds for $\mathcal{C}(\emptyset, \mathcal{A})$, by induction on the number $n$ of elements of $\mathcal{A}$. Since $\mathcal{A} \neq \emptyset$, we have $n>0$. The base case $n=1$ is the generalized Schönflies theorem, stating that an embedded tame $(d-1)$-sphere in $\mathbb{S}^{d}$ is the image of a hypersphere under a homeomorphism $\mathbb{S}^{d} \rightarrow \mathbb{S}^{d}$. Specifically, the complement of a pseudosphere is a disjoint union of two $d$-dimensional cells whose closures are balls.

Let $n>1$, and let $c$ be the closure of a connected component of $\mathcal{C}(\emptyset, \mathcal{A} \backslash\{n\})$. By induction on $n$, it is a cell of dimension $d$. The connected components of $S_{n} \cap c$ correspond to the closures of connected components of $\mathcal{C}(\emptyset, \mathcal{A} /\{n\})$. By the induction hypothesis, applied to the arrangement $\mathcal{A} /\{n\}$ of $n-1$ pseudospheres in $\mathbb{S}^{d-1}$, these are closed balls of dimension $d-1$ whose boundary lies in $\partial c$. Since $S_{n}$ is tame, $S_{n} \cap c$ is tame in $c$. Hence by the generalized Schönflies theorem, the closure of any connected component of $c \backslash S_{n}$ is a ball of dimension $d$. Note that this statement also holds when (in the non-uniform case) $S_{n} \cap c$ is contained in the boundary of $c$. In conclusion, the connected components of $\mathcal{C}(\emptyset, \mathcal{A})$ are cells of dimension $d$ whose closures are balls.

It remains to prove the theorem in the full rank case with $I \neq \emptyset$. We can assume $\mathcal{C}(I, \mathcal{A}) \neq \emptyset$. Since $\mathcal{A}$ is of full rank, $\mathcal{C}\left(E_{n}, \mathcal{A}\right)=\emptyset$, hence $I \neq E_{n}$. By Axiom (A1), $S_{I}$ is homeomorphic to some sphere $\mathbb{S}^{e}$. There is an $R \subset I$ with $|R| \leq d-e$ and $S_{R}=S_{I}$. The $\operatorname{set} \mathcal{C}(I, \mathcal{A})$ is mapped to $\mathcal{C}(\emptyset, \mathcal{A} / R)$ by the restriction of a homeomorphism $S_{I} \rightarrow \mathbb{S}^{e}$. It has already been proven that the closure of any connected component of $\mathcal{C}(\emptyset, \mathcal{A} / R)$ is a ball. Thus, the closure of any connected component of $\mathcal{C}(I, \mathcal{A})$ is a ball, as well. 
Note that the preceding theorem becomes wrong when dropping the hypothesis that pseudospheres are tame. In fact, there are wild 2-spheres in $\mathbb{S}^{3}$ (e.g., the famous Horned Sphere of Alexander). We consider arrangements of oriented embedded spheres (not necessarily tame) in Section 8 and prove that these wild arrangements have the same combinatorics as tame arrangements.

Let $\mathcal{A}=\left\{S_{1}, \ldots, S_{n}\right\}$ be an ordered multiset of oriented pseudospheres in $\mathbb{S}^{d}$. For $R \subset E_{n}$, denote $\mathcal{A}_{R}=\left\{S_{j} \mid j \in R\right\}$. In the remainder of this section we show that one can replace Axioms (A1) and (A2) by the following single Axiom ( $\mathbf{A}^{\prime}$ ):

(A') Let $R \subset E_{n}$ such that $S_{R^{\prime}} \neq S_{R}$ for any proper subset $R^{\prime}$ of $R$ (i.e., $R$ is an independent set). Then $\mathcal{A}_{R}$ is equivalent to an arrangement of $|R|$ oriented hyperspheres in $\mathbb{S}^{d}$.

Theorem 3. An ordered multiset $\mathcal{A}=\left\{S_{1}, \ldots, S_{n}\right\}$ of oriented pseudospheres in $\mathbb{S}^{d}$ satisfies Axiom $\left(A^{\prime}\right)$ if and only if it is an arrangement of oriented pseudospheres.

Proof. First, we assume that $\mathcal{A}$ satisfies Axiom ( $\left.\mathrm{A}^{\prime}\right)$ and prove that $\mathcal{A}$ is an arrangement of oriented pseudospheres. Let $\emptyset \neq R \subset E_{n}$, and assume that $S_{R} \neq \emptyset$. Replacing $R$ by a subset if necessary, we can assume that $S_{R^{\prime}} \neq S_{R}$ for any proper subset $R^{\prime}$ of $R$. By Axiom $\left(\mathrm{A}^{\prime}\right), \mathcal{A}_{R}$ is equivalent to an arrangement of oriented hyperspheres. Thus, $S_{R}$ is homeomorphic to a sphere, and Axiom (A1) holds. Let $i \in E_{n}$ with $S_{R} \not \subset S_{i}$; we wish to prove (A2). There is some $\tilde{R} \subset R \cup\{i\}$ with $S_{\tilde{R}}=S_{R \cup\{i\}}$, such that $S_{R^{\prime}} \neq S_{\tilde{R}}$ for any proper subset $R^{\prime}$ of $\tilde{R}$. Since, by Axiom $\left(\mathrm{A}^{\prime}\right), \mathcal{A}_{\tilde{R}}$ is equivalent to an arrangement of oriented hyperspheres, it follows that $\operatorname{dim} S_{\tilde{R} \backslash\{i\}}=\operatorname{dim} S_{R \cup\{i\}}+1$. Since both $S_{R}$ and $S_{R \cup\{i\}}$ are spheres by Axiom (A1) (that has already been proven) and since $S_{R} \not \subset S_{i}$ by hypothesis, it follows that

$$
\operatorname{dim} S_{R \cup\{i\}}+1=\operatorname{dim} S_{\tilde{R} \backslash\{i\}} \geq \operatorname{dim} S_{R}>\operatorname{dim} S_{R \cup\{i\}} .
$$

Thus, $S_{\tilde{R} \backslash\{i\}} \subset S_{R}$ is a pair of spheres of the same dimension $\operatorname{dim} S_{R \cup\{i\}}+1$, hence, $S_{\tilde{R} \backslash\{i\}}=S_{R}$. Since $\mathcal{A}_{\tilde{R}}$ is equivalent to an arrangement of oriented hyperspheres, Axiom (A2) holds for $S_{\tilde{R} \backslash\{i\}}=S_{R}$ and $S_{i}$. In conclusion, $\mathcal{A}$ is an arrangement of oriented pseudospheres.

Secondly, we assume that $\mathcal{A}$ is an arrangement of oriented pseudospheres and prove by induction on $|R|$ that ( $\left.\mathrm{A}^{\prime}\right)$ holds. If $|R|=1$, then $\left(\mathrm{A}^{\prime}\right)$ is nothing but the generalized Schönflies theorem. In the general case, let $\emptyset \neq R \subset E_{n}$ such that $S_{R^{\prime}} \neq S_{R}$ for any proper subset $R^{\prime}$ of $R$, and let $i \in R$. It easily follows that $S_{R^{\prime}} \neq S_{R \backslash\{i\}}$ for any proper subset $R^{\prime}$ of $R \backslash\{i\}$. By induction, $\mathcal{A}_{R \backslash\{i\}}$ satisfies Axiom ( $\left.\mathrm{A}^{\prime}\right)$, and we can assume that $\mathcal{A}_{R \backslash\{i\}}$ is an arrangement of oriented hyperspheres.

Let $H \subset \mathbb{S}^{d}$ be a hypersphere that does not contain $S_{R \backslash\{i\}}$. Recall that the connected components of $\mathcal{C}\left(I, \mathcal{A}_{R \backslash\{i\}}\right)$ are topological cells, for any proper subset $I$ of $R \backslash\{i\}$. Our aim is to transform $S_{i}$ into $H$, fixing $\mathcal{A}_{R \backslash\{i\}}$ cellwise, which implies Axiom (A') for $\mathcal{A}_{R}$. By Axiom (A2) and by the generalized Schönflies theorem, $S_{R \backslash\{i\}} \cap S_{i}$ can be mapped to $S_{R \backslash\{i\}} \cap H$ by some orientation preserving homeomorphism $S_{R \backslash\{i\}} \rightarrow S_{R \backslash\{i\}}$. The homeomorphism can be extended to a homeomorphism $\mathbb{S}^{d} \rightarrow \mathbb{S}^{d}$ fixing all cells of $\mathcal{A}_{R \backslash\{i\}}$, by the cone construction [23]. 
We now proceed with transforming $S_{i}$ in cells of $\mathcal{A}_{R \backslash\{i\}}$ of higher dimension. Let $R^{\prime}$ be a proper subset of $R \backslash\{i\}$. By induction on $\left|R \backslash R^{\prime}\right|$, we can assume that $S_{R^{\prime} \cup\{j\}} \cap S_{i}=$ $S_{R^{\prime} \cup\{j\}} \cap H$, for all $j \in R \backslash\left(R^{\prime} \cup\{i\}\right)$. Let $C \subset S_{R^{\prime}}$ be the closure of a cell of $\mathcal{A}_{R \backslash\{i\}}$ with $\operatorname{dim} C=\operatorname{dim} S_{R^{\prime}}$. It follows from Axiom (A2) that $B=S_{i} \cap C$ is a tame ball in $C$. By the generalized Schönflies theorem and since $\partial B \subset H$, we can map $C \cap S_{i}$ to $C \cap H$ by an orientation preserving homeomorphism $C \rightarrow C$ that is the identity on $\partial C$. It can be extended to a homeomorphism $\mathbb{S}^{d} \rightarrow \mathbb{S}^{d}$ by the cone construction, fixing all cells of $\mathcal{A}_{R \backslash\{i\}}$. Thus, we can transform $S_{R^{\prime}} \cap S_{i}$ into $S_{R^{\prime}} \cap H$. Finally, when we achieve $R^{\prime}=\emptyset$, we have $S_{R^{\prime}} \cap S_{i}=S_{i}=H$. Therefore, $\mathcal{A}_{R}$ is equivalent to an arrangement of oriented hyperspheres. This proves $\left(\mathrm{A}^{\prime}\right)$, as claimed.

Corollary 1. If an arrangement $\mathcal{A}$ of oriented pseudospheres in $\mathbb{S}^{d}$ is of full rank and $n=d+1$, then $\mathcal{A}$ is equivalent to $\mathcal{A}(d,+)$ or $\mathcal{A}(d,-)$.

Proof. Let $R \subset E_{d+1}$ be minimal such that $\mathcal{A}_{R}$ is of full rank. By the preceding theorem, $\mathcal{A}_{R}$ is equivalent to an arrangement of oriented hyperspheres of full rank. Such an arrangement consists of at least $d+1$ hyperspheres, hence $R=E_{d+1}$ and $\mathcal{A}_{R}=\mathcal{A}$.

Any arrangement of $d+1$ oriented hyperspheres of full rank is dual to an arrangement of $d+1$ unit vectors in $\mathbb{R}^{d+1}$ that span $\mathbb{R}^{d+1}$. Since $G L_{d+1}(\mathbb{R})$ acts transitively on those vector arrangements, it follows that $\mathcal{A}$ is equivalent to $\mathcal{A}(d,+)$ or $\mathcal{A}(d,-)$, depending on its orientation.

\section{Hyperline Sequences Associated to Arrangements of Oriented Pseudospheres}

The aim of our paper is to prove the TRT in the setting of hyperline sequences, i.e., to establish a one-to-one correspondence between oriented matroids and equivalence classes of arrangements of oriented pseudospheres. In this section we settle one direction of this correspondence. We associate to any arrangement of oriented pseudospheres an oriented matroid given by hyperline sequences, compatible with deletions and contractions.

We first expose the geometric idea. By a cycle of an arrangement $\mathcal{A}$ of oriented pseudospheres in $\mathbb{S}^{d}$, we mean an embedded circle $\mathbb{S}^{1} \subset \mathbb{S}^{d}$ that is the intersection of some elements of $\mathcal{A}$. Let $L$ be a cycle of $\mathcal{A}$ with a choice of an orientation. It corresponds to a hyperline sequence $(Y \mid Z)$, as follows. The positively oriented bases of $Y$ correspond to $(d-1)$-tuples of pseudospheres containing $L$ inducing the correct orientation on $L$. The set of all elements of $\mathcal{A}$ containing $L$ corresponds to $E(Y)$. The zero-dimensional cells of $\mathcal{A}$ on $L$ occur in a cyclic order, corresponding to the cyclic order of $Z$. Let $S_{e} \in \mathcal{A}$. If in a point of $L \cap S_{e}$ the cycle $L$ passes from the negative side of $S_{e}$ to the positive side, then we have an element $e$ in the corresponding atom of $(Y \mid Z)$ (see Fig. 1). Furthermore, in the second point of $L \cap S_{e}$, the cycle $L$ passes from the positive to the negative side, yielding an element $\bar{e}$ in the atom that is opposite to the first atom.

The meaning of Axiom (H3) in the setting of arrangements of oriented pseudospheres is that any two cycles in any rank 3 contraction of $\mathcal{A}$ have non-empty intersection. Figure 2 provides a visualization of Axiom (H4) in an arrangement of three oriented pseudospheres in $\mathbb{S}^{2}$ : if we get from the pseudosphere $k$ the cyclic order $(\{i\},\{j\},\{\bar{l}\},\{\bar{J}\})$, then 


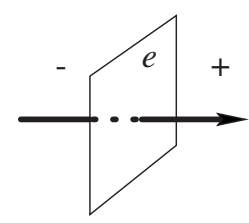

Fig. 1. The atom $\{e\}$ on a cycle.

we get the cyclic order $(\{\bar{k}\},\{j\},\{k\},\{\bar{J}\})$ from $i$ and the cyclic order $(\{\bar{k}\},\{\bar{l}\},\{k\},\{i\})$ from $j$.

We formalize this idea in the rest of this section. Let $\mathcal{A}=\left\{S_{1}, \ldots, S_{n}\right\}$ be an arrangement of $n$ oriented pseudospheres of full rank in $\mathbb{S}^{d}$. Our aim is to associate to $\mathcal{A}$ an oriented matroid $X(\mathcal{A})$ of rank $d+1$ over $E_{n}$. If $d=0$ then define

$$
X(\mathcal{A})=\left\{e \in E_{n} \mid S_{e}^{+}=\{+1\}\right\} \cup\left\{\bar{e} \in \overline{E_{n}} \mid S_{e}^{+}=\{-1\}\right\},
$$

which is obviously an oriented matroid over $E_{n}$ of rank 1 .

In the case $d=1$, the orientation of $\mathbb{S}^{1}$ yields a cyclic order $p_{0}, p_{1}, \ldots, p_{2 k-1}$ on the points of $S_{1} \cup \ldots \cup S_{n}$. For $a \in\{0, \ldots, 2 k-1\}$, we define $X^{a} \subset \mathbf{E}_{\mathbf{n}}$ by

(1) $e \in X^{a}$ if $p_{a} \in S_{e}$ and, along the cyclic orientation of $\mathbb{S}^{1}$, one passes in $p_{a}$ from $S_{e}^{-}$to $S_{e}^{+}$, and

(2) $\bar{e} \in X^{a}$ if $p_{a} \in S_{e}$ and one passes in $p_{a}$ from $S_{e}^{+}$to $S_{e}^{-}$.

It is easy to check that $\left(X^{0}, \ldots, X^{2 k-1}\right)$ yields a hyperline sequence of rank 2 over $E_{n}$.

In the case $d=2$, let $\gamma \subset \mathbb{S}^{2}$ be an oriented cycle of $\mathcal{A}$. Let $R \subset E_{n}$ be the indices of oriented pseudospheres containing $\gamma$. There is an oriented matroid $Y(\gamma)$ of rank 1 over $R$, with $e \in Y(\gamma)$ (resp. $\bar{e} \in Y(\gamma)$ ) if the orientation of $S_{e}$ coincides (resp. does not coincide) with the orientation of $\gamma$. As in the preceding paragraph, we obtain a hyperline sequence $Z(\gamma)$ of rank 2 over $E_{n} \backslash R$. We collect the pairs $(Y(\gamma) \mid Z(\gamma))$ to form a set $X(\mathcal{A})$, where $\gamma$ runs over all oriented cycles of $\mathcal{A}$. Axioms (H1) and (H2) are obvious for $X(\mathcal{A})$. To prove Axiom (H3), let $\left[x_{1}, x_{2}, x_{3}\right]$ and $\left[y_{1}, y_{2}, y_{3}\right]$ be two positively oriented bases of $X(\mathcal{A})$. By definition, the pseudospheres $S_{y_{1}^{*}}, S_{y_{2}^{*}}, S_{y_{3}^{*}}$ have no point in common. Specifically, one of them, say, $S_{y_{1}^{*}}$, does not contain $S_{x_{1}^{*}} \cap S_{x_{2}^{*}}$. Thus $S_{y_{1}^{*}}$ intersects $S_{x_{1}^{*}}$ transversely in $S_{x_{1}^{*}} \backslash S_{x_{2}^{*}}$, hence $\left[x_{1}, x_{2}, y_{1}\right]$ or $\left[x_{1}, x_{2}, \overline{y_{1}}\right]$ is a positively oriented base of $X(\mathcal{A})$. It remains to prove Axiom (H4). Here we use the Jordan-Schönflies theorem [20], stating that the complement of an embedded 1-sphere in $\mathbb{S}^{2}$ is a disjoint union of two discs. With this in mind, Axiom (H4) can be read off from Fig. 2.

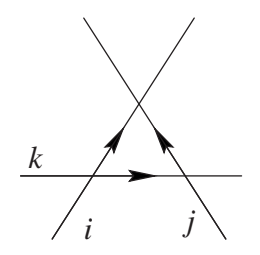

Fig. 2. Axiom (H4) in rank 3. 
In the case $d \geq 3$, let $\gamma$ be an oriented cycle of $\mathcal{A}$. Let $R_{\gamma}=\left\{r \in E_{n} \mid \gamma \subset S_{r}\right\}$. As in the preceding paragraph, the cyclic orientation of $\gamma$ induces a cyclic order of the oriented points

$$
\gamma \cap \bigcup_{e \in E_{n} \backslash R_{\gamma}} S_{e},
$$

yielding a hyperline sequence $Z(\gamma)$ of rank 2 over $E_{n} \backslash R_{\gamma}$. Since $\mathcal{A}$ is of full rank, there are $i, j \in E_{n}$ so that $S_{i} \cap S_{j}$ is a sphere of dimension $d-2$ disjoint from $\gamma$. We may assume that $Z(\gamma)$ yields the cyclic order $(\{i\},\{j\},\{\bar{l}\},\{\bar{J}\})$, by changing the roles of $i$ and $j$ if necessary. Then

$$
\mathcal{A}(i, j)=((\mathcal{A} /\{i\}) /\{j\})_{R_{\gamma}}
$$

is an arrangement of oriented pseudospheres in $\mathbb{S}^{d-2}$ of full rank over $R_{\gamma}$, and by induction it corresponds to an oriented matroid $Y(i, j)$ of rank $d-1$ over $R_{\gamma}$.

We show that $Y(i, j)$ does not depend on the choice of $i, j$. By symmetry of $i$ and $j$, it suffices to pick $k \in E_{n}$ so that $S_{i} \cap S_{k}$ is a sphere of dimension $d-2$ disjoint from $\gamma$ and $Z(\gamma)$ yields the cyclic order $(\{i\},\{k\},\{\bar{l}\},\{\bar{k}\})$, and to show that $Y(i, j)=Y(i, k)$. We will prove that the cyclic order of signed points on oriented cycles of $\mathcal{A}(i, j)$ coincides with those of $\mathcal{A}(i, k)$. It is clear that this implies $Y(i, j)=Y(i, k)$.

We choose $R \subset R_{\gamma}$ so that $S_{R} \cap S_{i} \cap S_{j}$ is a cycle of $\mathcal{A}(i, j)$, and consider the 2-sphere $S=S_{R} \cap S_{i}$. Both $s_{j}=S \cap S_{j}$ and $s_{k}=S \cap S_{k}$ are embedded 1-spheres in $S$ that are either equal or intersect in two points. Both $s_{j}$ and $s_{k}$ have the positive (resp. negative) point of $\gamma \cap S$ on their positive (resp. negative) side. Thus, again using the Jordan-Schönflies theorem, the situation is as in Fig. 3. Let $C$ be a connected component of $S \backslash\left(s_{j} \cup s_{k}\right)$ disjoint from $\gamma$. Let $a, b \in R_{\gamma} \backslash R$. Since $s_{a}=S \cap S_{a}$ is equal to $s_{b}=S \cap S_{b}$ or $s_{a}$ intersects $s_{b}$ transversely in $S \cap \gamma$, it follows that $C \cap s_{a}$ and $C \cap s_{b}$ are parallel arcs. Hence, the cyclic order of the four signed points of $s_{j} \cap\left(s_{a} \cup s_{b}\right)$ coincides with the order on $s_{k} \cap\left(s_{a} \cup s_{b}\right)$. This proves our claim $Y(i, j)=Y(i, k)$. Since $Y(i, j)$ does not depend on the choice of $i$ and $j$, we denote $Y(\gamma)=Y(i, j)$.

Now, we define $X(\mathcal{A})$ as the set of all pairs $(Y(\gamma) \mid Z(\gamma))$, where $\gamma$ runs over all oriented cycles of $\mathcal{A}$, each cycle occurring in both orientations. It remains to show that $X(\mathcal{A})$ is an oriented matroid of rank $d+1$ over $E_{n}$. First, $X(\mathcal{A})$ is not empty. Axiom (H1) is trivial. Any subarrangement of $d-1$ pseudospheres defines a cycle $\gamma$ of $\mathcal{A}$, up to

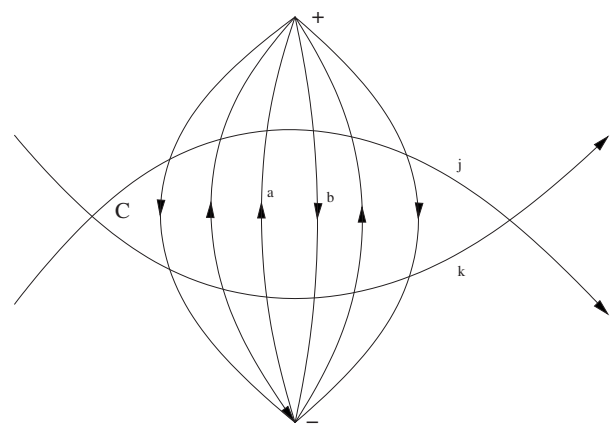

Fig. 3. Parallel cycles. 
orientation. Hence Axiom (H2) follows from $Y(\gamma)=-Y(-\gamma)$ and $Z(\gamma)=-Z(-\gamma)$. Axioms (H3) and (H4) follow from the corresponding axioms in the case $d=2$, since we can argue by contraction onto a 2 -sphere containing the two cycles of $\mathcal{A}$ involved in Axioms (H3) and (H4).

In conclusion, $X(\mathcal{A})$ is an oriented matroid. By Theorem 1 , we can define $\chi(\mathcal{A})$ as the chirotope that has the same positively oriented bases as $X(\mathcal{A})$. Let $R \subset E_{n}$ so that $\mathcal{A} \backslash R$ (resp. $\mathcal{A} / R$ ) is an arrangement of oriented pseudospheres of full rank. It is easy to see that $\chi(\mathcal{A} \backslash R)=\chi(\mathcal{A}) \backslash R($ resp. $\chi(\mathcal{A} / R)=\chi(\mathcal{A}) / R)$.

\section{The Topological Representation Theorem-Statement and Base Cases}

Theorem 4 (Topological Representation Theorem). To anyoriented matroid X of rank $r$ over $E_{n}$, there is an arrangement $\mathcal{A}(X)$ of $n$ oriented pseudohyperspheres in $\mathbb{S}^{r-1}$ of full rank with $X=X(\mathcal{A}(X))$. The equivalence class of $\mathcal{A}(X)$ is unique.

We prove Theorem 4 by induction on the number of elements and the rank of $X$. In this section we prove the base cases $r \leq 2$ and $n=r$. The next section is devoted to the inductive step.

Claim 6.1. Theorem 4 holds for $r=1$.

Proof. Let $X$ be an oriented matroid of rank 1 over $E_{n}$. An oriented pseudosphere $S$ in $\mathbb{S}^{0}=\{+1,-1\}$ is the empty set, together with the information whether $S^{+}=\{+1\}$ or $S^{+}=\{-1\}$. For $i \in E_{n}$, set $S_{i}^{+}=\{+1\}$ if $i \in X$ and $S_{i}^{+}=\{-1\}$ otherwise. It is obvious that $\mathcal{A}(X)=\left\{S_{1}, \ldots, S_{n}\right\}$ is an arrangements of oriented pseudospheres such that $X(\mathcal{A}(X))=X$, and that $\mathcal{A}(X)$ is unique with this property.

Claim 6.2. Theorem 4 holds for $r=2$.

Proof. Let $X$ be a hyperline sequence of rank 2 over $E_{n}$. The hyperline sequence $X$ is a map from some cyclic group $C_{2 k}$ to non-empty subsets of $\mathbf{E}_{n}$. We consider $C_{2 k}$ as a subgroup of $\mathbb{S}^{1}$. An oriented pseudosphere corresponds to an embedding of $\mathbb{S}^{0}=\{+1,-1\}$ into $\mathbb{S}^{1}$. For $i \in E_{n}$, let $a_{i} \in C_{2 k}$ so that $i \in X^{a}$. Define $\psi_{i}(+1)=a_{i} \in \mathbb{S}^{1}$ and $\psi_{i}(-1)=-a_{i} \in \mathbb{S}^{1}$. It follows easily that $\mathcal{A}(X)=\left\{S_{1}, \ldots, S_{n}\right\}$ is an arrangements of oriented pseudospheres such that $X(\mathcal{A}(X))=X$, and that $\mathcal{A}(X)$ is unique with this property.

Lemma 1. There are exactly two chirotopes of rank $|E|$ over $E$, namely one with $[1, \ldots, r]$ as a positively oriented base, and the other with $[1, \ldots, r-1, \bar{r}]$ as a positively oriented base.

Proof. Set $r=|E|$. Without loss of generality, let $\chi$ be a chirotope of rank $r$ over $E_{r}=E$. There are exactly two equivalence classes of oriented $(r-1)$-simplices in $E_{r}$, 
namely those equivalent to $[1, \ldots, r]$ and those equivalent to $[1, \ldots, r-1, \bar{r}]$. Thus, by Axiom $(\mathrm{C} 2), \chi$ is completely determined by $\chi([1, \ldots, r])$. Axiom $(\mathrm{C} 1)$ implies $\chi([1, \ldots, r])= \pm 1$. Hence there are at most two chirotopes of rank $|E|$ over $E$. The conditions in Axioms (C3) and (C4) are empty for $|E|=r$. Thus, there are two chirotopes of $\operatorname{rank}|E|$ over $E$.

Claim 6.3. Theorem 4 holds for $n=r$.

Proof. We prove the claim in the setting of chirotopes. Let $\chi$ be a chirotope of rank $r$ over $E_{r}$. By Lemma $1, \chi$ is determined by whether $[1, \ldots, r]$ is a positively oriented base of $\chi$ or not. In the former case, define $\mathcal{A}(\chi)=\mathcal{A}(r-1,+)$; in the latter case, define $\mathcal{A}(\chi)=\mathcal{A}(r-1,-)$. We have $\chi(\mathcal{A}(\chi))=\chi$ by construction, and the uniqueness of $\mathcal{A}(\chi)$ follows from Corollary 1.

\section{The Topological Representation Theorem-General Case}

This section is devoted to the inductive step in the proof of Theorem 4. Let $n>r>2$. Suppose that Theorem 4 holds for all oriented matroids of rank $r$ with less than $n$ elements and for all oriented matroids of rank less than $r$. Thus, for any non-empty $R \subset E_{n}$ if the contraction $X / R$ (resp. the deletion $X \backslash R$ ) is defined, then there is an essentially unique arrangement $\mathcal{A}(X / R)$ (resp. $\mathcal{A}(X \backslash R)$ ) of oriented pseudospheres in $\mathbb{S}^{r-1-|R|}$ (resp. in $\left.\mathbb{S}^{r-1}\right)$ of full rank with $X / R=X(\mathcal{A}(X / R))$ (resp. with $X \backslash R=X(\mathcal{A}(X \backslash R))$ ).

There is some element of $X$, say, $n$ for simplicity, such that the deletion $X \backslash\{n\}$ is an oriented matroid of rank $r$. Denote $\left\{S_{1}, \ldots, S_{n-1}\right\}=\mathcal{A}(X \backslash\{n\})$. Our aim is to construct an oriented pseudosphere $S_{n} \subset \mathbb{S}^{r-1}$ as the image of a tame embedding $\psi: \mathbb{S}^{r-2} \rightarrow \mathbb{S}^{r-1}$, so that $\left\{S_{1}, \ldots, S_{n}\right\}$ is an arrangement of oriented pseudospheres with $X\left(\left\{S_{1}, \ldots, S_{n}\right\}\right)$ $=X$.

We outline informally the idea of the construction of $\psi$. We start with the arrangement $\mathcal{A}(X /\{n\})$ in $\mathbb{S}^{r-2}$. We require that $\psi$ maps this arrangement "consistently" to the arrangement $\mathcal{A}(X \backslash\{n\})$, in the sense that any cell in $\mathcal{C}(I, \mathcal{A}(X /\{n\}))$ is mapped to a cell in $\mathcal{C}(I, \mathcal{A}(X \backslash\{n\}))$ in the correct orientation. It turns out that this forces $\left\{S_{1}, \ldots, S_{n}\right\}$ to be an arrangement of oriented pseudospheres. Moreover, we show that if $S_{n}$ intersects the cycles of $\mathcal{A}(X \backslash\{n\})$ in a way consistent with the rank 2 contractions of $X$ (i.e., the cyclic order on its hyperline sequences), then $X\left(\left\{S_{1}, \ldots, S_{n}\right\}\right)=X$. Our construction of $\psi$ is iterative. We start with defining $\psi$ on zero-dimensional cells of $\mathcal{A}(X /\{n\})$ and show that if it is defined on $d$-dimensional cells then it can be consistently extended to $(d+1)$-dimensional cells. It turns out that this is possible in an essentially unique way.

Let us formalize this idea. By induction hypothesis, the arrangement $\mathcal{A}(X /\{n\})$ exists and is unique up to equivalence. For any element $i \in E(X /\{n\})$ of $X /\{n\}$, we denote by $s_{i}$ the oriented pseudosphere of $\mathcal{A}(X /\{n\})$ that corresponds to $i$. For any $R \subset E(X /\{n\})$, set $s_{R}=\mathbb{S}^{r-2} \cap \bigcap_{j \in R} s_{j}$, and similarly $S_{R}=\mathbb{S}^{r-1} \cap \bigcap_{j \in R} S_{j}$ for $R \subset E_{n-1}$. Recall that the $d$-dimensional skeleton $\mathcal{A}^{(d)}$ of an arrangement $\mathcal{A}$ of oriented pseudospheres is the union of its cells of dimension $\leq d$. For any $R \subset E_{n}$, define $R / n=R \cap E(X /\{n\})$. 
Definition 7. Let $t<r$. A $t$-admissible embedding is an embedding

$$
\psi^{(t)}:(\mathcal{A}(X /\{n\}))^{(t-1)} \rightarrow \mathbb{S}^{r-1}
$$

such that for any $R \subset E_{n-1}$ with $\operatorname{dim} s_{R / n} \leq t-1$ it holds that

(1) $\psi^{(t)}\left(s_{R / n}\right)=S_{R}$ or $\psi^{(t)}\left(s_{R / n}\right)$ is a pseudosphere in $S_{R}$,

(2) if $\psi^{(t)}\left(s_{R / n}\right) \neq S_{R}$, then any cycle of $\mathcal{A}(X \backslash\{n\})$ in $S_{R}$ is either contained in $\psi^{(t)}\left(s_{R / n}\right)$ or meets both connected components of $S_{R} \backslash \psi\left(s_{R / n}\right)$, and

(3) for any $i \in E(X /\{n\}) \backslash R$ it holds that $\psi\left(s_{R / n} \cap s_{i}^{+}\right) \subset S_{R} \cap S_{i}^{+}$and $\psi\left(s_{R / n} \cap s_{i}^{-}\right) \subset$ $S_{R} \cap S_{i}^{-}$.

By the following two lemmas, in our request for the pseudosphere $S_{n}$ it suffices to study $(r-1)$-admissible embeddings.

Lemma 2. Let $\psi: \mathbb{S}^{r-2} \rightarrow \mathbb{S}^{r-1}$ be a tame embedding that defines an oriented pseudosphere $S_{n}$. If $\psi$ is $(r-1)$-admissible then $\mathcal{A}=\left\{S_{1}, \ldots, S_{n}\right\}$ is an arrangement of oriented pseudospheres.

Proof. We prove that $\mathcal{A}=\left\{S_{1}, \ldots, S_{n}\right\}$ satisfies Axioms (A1) and (A2):

(A1) Let $R \subset E_{n}$. We have to show that $S_{R}$ is empty or homeomorphic to a sphere. If $n \notin R$ then we are done since $\left\{S_{1}, \ldots, S_{n-1}\right\}$ is an arrangement. If $n \in R$ then we have $S_{R}=\psi\left(s_{R / n}\right)$, and $s_{R / n}$ is empty or homeomorphic to a sphere since $\mathcal{A}(X /\{n\})$ is an arrangement.

(A2) Let $R \subset E_{n}$ and $i \in E_{n}$ with $S_{R} \not \supset S_{i}$. Since $\psi$ is $(r-1)$-admissible, $S_{R} \cap S_{i}$ is a pseudosphere in $S_{R}$. It remains to show that $S_{R} \cap S_{i}^{+}$and $S_{R} \cap S_{i}^{-}$are both non-empty. If $R \cup\{i\} \subset E_{n-1}$ then we are done, since $\left\{S_{1}, \ldots, S_{n-1}\right\}$ is an arrangement of oriented pseudospheres.

If $n \in R$ then $S_{R}=\psi\left(s_{R / n}\right)$ and $S_{R} \cap S_{i}=\psi\left(s_{(R \cup\{i\}) / n}\right)$. Since $S_{R} \not \supset S_{i}$, we have $s_{R / n} \not \supset s_{i}$. Thus in this case, Axiom (A2) for $\mathcal{A}$ follows from Axiom (A2) for $\mathcal{A}(X /\{n\})$ applied to $s_{R / n}$ and $s_{i}$, since $\psi\left(s_{R / n} \cap s_{i}^{+}\right) \subset S_{R} \cap S_{i}^{+}$and $\psi\left(s_{R / n} \cap s_{i}^{-}\right) \subset S_{R} \cap S_{i}^{-}$.

If $n=i$, then $R=R / n$ since otherwise $S_{n}=S_{i} \subset S_{R}$ by definition of $S_{n}$. Hence $S_{R} \cap S_{n}=\psi\left(s_{R}\right)$ is a pseudosphere in $S_{R}$. Since $\mathcal{A} \backslash\{n\}$ is of full rank, $S_{R}$ contains a cycle of $\mathcal{A} \backslash\{n\}$. By the second property in the definition of $(r-1)$-admissible embeddings, applied to the empty set, this circle meets both connected components of $\mathbb{S}^{r-1} \backslash S_{n}$, which implies Axiom (A2).

Thus, $\mathcal{A}$ is an arrangement of oriented pseudospheres.

Lemma 3. Let $\tilde{\psi}: \mathbb{S}^{r-2} \rightarrow \mathbb{S}^{r-1}$ be a tame embedding that defines an oriented pseudosphere $\tilde{S}_{n}$. Assume that $\tilde{\mathcal{A}}=\left\{S_{1}, \ldots, S_{n-1}, \tilde{S}_{n}\right\}$ is an arrangement of oriented pseudospheres. If $X(\tilde{\mathcal{A}})=X$ then there is an orientation preserving homeomorphism $\varphi: \mathbb{S}^{r-2} \rightarrow \mathbb{S}^{r-2}$ such that $\tilde{\psi} \circ \varphi$ is $(r-1)$-admissible.

Proof. The arrangement $\tilde{\mathcal{A}} /\{n\}$ of oriented pseudospheres is given by the pre-images of $S_{1}, \ldots, S_{n-1}$ under $\tilde{\psi}$. By the induction hypothesis in the proof of the TRT, the 
equivalence class of $\mathcal{A}(X /\{n\})$ is determined by the property $X(\mathcal{A}(X /\{n\}))=X /\{n\}$. If $X(\tilde{\mathcal{A}})=X$, then $X(\tilde{\mathcal{A}} /\{n\})=X /\{n\}$. Hence, the arrangement $\mathcal{A}(X /\{n\})$ is equivalent to $\tilde{\mathcal{A}} /\{n\}$. Let $\varphi: \mathbb{S}^{r-2} \rightarrow \mathbb{S}^{r-2}$ be the orientation preserving homeomorphism realizing this equivalence. It easily follows that $\tilde{\psi} \circ \varphi$ is $(r-1)$-admissible.

According to the preceding two lemmas, we shall construct $S_{n}$ via a tame $(r-1)$ admissible embedding. Moreover, we need to take into account the rank 2 contractions of $X$, as follows. Let $\psi^{(1)}$ be a 1 -admissible embedding. Assume that for any contraction $X / R$ of rank 2 with $n \in E(X / R)$, the oriented zero-dimensional sphere $\psi\left(s_{R}\right)$ extends the arrangement of oriented pseudospheres on the oriented cycle $S_{R}$ that is induced by $\mathcal{A}(X \backslash\{n\})$ to an arrangement equivalent to $\mathcal{A}(X / R)$. Then we call $\psi^{(1)}$ compatible with $X$. In the next three lemmas, we prove that there is an essentially unique $(r-1)$-admissible embedding whose restriction to the 0 -skeleton of $\mathcal{A}(X /\{n\})$ is compatible with $X$.

Lemma 4. There is a tame 1-admissible embedding $\psi^{(1)}$ that is compatible with X. It is unique up to composition with a homeomorphism $(\mathcal{A}(X \backslash\{n\}))^{(1)} \rightarrow(\mathcal{A}(X \backslash\{n\}))^{(1)}$ that fixes $(\mathcal{A}(X \backslash\{n\}))^{(0)}$.

Proof. Let $R \subset E_{n-1}$ such that $X / R$ is of rank 2. We first prove the uniqueness of $\psi^{(1)}$. If $s_{R / n} \approx \mathbb{S}^{0}$, then the cyclic order of the signed elements of $X / R$ determines in which cells of $\mathcal{A}(X \backslash\{n\})$ on $S_{R}$ the two points of $s_{R}$ must be mapped to, provided $\psi^{(1)}$ is compatible with $X$. If they are mapped to zero-dimensional cells of $\mathcal{A}(X \backslash\{n\})$ then their image is unique. If they are mapped to one-dimensional cells, then their image is unique up to a homeomorphism of these cells fixing the boundary.

If $\operatorname{dim} s_{R / n}>0$, then $R \neq R / n$, and $s_{R / n}$ is a cycle of $\mathcal{A}(X /\{n\})$. Any 0 -cell on $s_{R / n}$ is contained in some pseudosphere $s_{i}$ of $\mathcal{A}(X /\{n\})$ that intersects $s_{R / n}$ transversely. Then $s_{R / n} \cap s_{i} \approx \mathbb{S}^{0}$, and if $\psi^{(1)}$ is 1-admissible then $\psi\left(s_{R / n} \cap s_{i}\right) \subset S_{R} \cap S_{i} \approx \mathbb{S}^{0}$. Moreover, if $\psi^{(1)}$ is 1-admissible then the intersection of $s_{R / n} \cap s_{i}$ with one side of a pseudosphere $s_{j}$ is mapped to the corresponding side of $S_{j}$. This determines the image of the two points of $s_{R / n} \cap s_{i}$ under $\psi^{(1)}$.

We prove the existence of $\psi^{(1)}$. According to the preceding two paragraphs, for any zero-dimensional cell $p$ of $\mathcal{A}(X /\{n\})$, a candidate for $\psi^{(1)}(p)$ is given by the cyclic order of the rank 2 contractions of $X$. We must ensure that the candidate does not depend on the choice of the contraction. Since any two cycles of $\mathcal{A}(X \backslash\{n\})$ are contained in some 2-sphere that is the intersection of pseudospheres in $\mathcal{A}(X \backslash\{n\})$, we can assume by a contraction that $X$ is of rank 3 . Then the pseudospheres $S_{1}, \ldots, S_{n-1}$ are cycles.

Let $i, j \in E_{n-1}$. If $i \notin E(X /\{n\})$ then $X /\{i\}= \pm X /\{n\}$, hence, the cyclic order of signed points on $S_{i}$ is a copy of $\mathcal{A}(X /\{n\})$, possibly with opposite orientation. In this case (and similarly if $j \notin E(X /\{n\}))$ it is easy to show that the candidates for $\psi^{(1)}$ imposed by $i$ and $j$ coincide. The case $i, j \in E(X /\{n\})$ with $s_{i} \cap s_{j} \neq \emptyset$ remains. The positive point $p$ of the oriented pseudosphere $s_{i}$ shall be mapped into the cell $C_{i}$ of $\mathcal{A}(X \backslash\{n\})$ on $S_{i}$ that corresponds to the atom of $X /\{i\}$ containing $\bar{n}$. If $(X /\{n\}) /\{i\}=(X /\{n\}) /\{j\}$ (resp. $(X /\{n\}) /\{i\}=-(X /\{n\}) /\{j\})$, then $p$ is the positive (resp. negative) point of $s_{j}$, thus shall be mapped into the cell $C_{j}$ on $S_{j}$ that corresponds to the atom of $X /\{j\}$ containing $\bar{n}$ (resp. containing $n$ ). 
If $X /\{i\}= \pm X /\{j\}$ then obviously $C_{i}=C_{j}$. Otherwise, $S_{i} \cap S_{j}$ is a 0-sphere containing both $C_{i}$ and $C_{j}$. Up to symmetry, we can assume that $(X /\{i\}) /\{n\}=(X /\{i\}) /\{j\}$, which means that the atom of $X /\{i\}$ containing $\bar{n}$ does also contain $\bar{J}$. Hence, $C_{i}$ corresponds to the atom of $X /\{j\}$ containing $i$. This atom also contains $\bar{n}$ (resp. $n$ ) if and only if

$$
(X /\{j\}) /\{n\}=-(X /\{j\}) /\{i\}=(X /\{i\}) /\{j\}=(X /\{i\}) /\{n\}
$$

(resp. $(X /\{j\}) /\{n\}=-(X /\{i\}) /\{n\})$. Thus $C_{i}=C_{j}$ by construction of $C_{i}$ and $C_{j}$. In conclusion, the candidates for $\psi^{(1)}(p)$ imposed by $i$ and $j$ coincide, which is enough to prove the existence of $\psi^{(1)}$.

Lemma 5. Let $t<r-1$, and let $\psi^{(t)}$ be a t-admissible embedding. For any $t-$ dimensional cell $c$ of $\mathcal{A}(X /\{n\})$ there is a cell $c^{\prime}$ of $\mathcal{A}(X \backslash\{n\})$ of dimension $t$ or $t+1$ such that $\psi^{(t)}(\partial c) \subset \partial c^{\prime}$.

Proof. Let $R \subset E_{n-1}$ be maximal so that $c \subset s_{R / n}$. In particular, $\operatorname{dim} s_{R / n}=\operatorname{dim} c=t$ and $\operatorname{dim} S_{R} \leq \operatorname{dim} s_{R / n}+1=t+1$. For any cell $b$ of dimension $\operatorname{dim} b=\operatorname{dim} c-1$ in $\partial c$ and any $j_{b} \in E(X /\{n\})$ with $b \subset s_{R / n} \cap s_{j_{b}}$, we have $\psi^{(t)}(b) \subset S_{R} \cap S_{j_{b}}$ since $\psi^{(t)}$ is $t$-admissible. By consequence, $\psi^{(t)}(\partial c) \subset S_{R}$.

Since $c$ is a cell of $\mathcal{A}(X /\{n\}), \partial c \cap s_{j}^{+}=\emptyset$ or $\partial c \cap s_{j}^{-}=\emptyset$, for all $j \in E(X /\{n\})$. Since $\psi^{(t)}$ is $t$-admissible, $\psi^{(t)}(\partial c) \cap S_{j}^{+}=\emptyset$ or $\psi^{(t)}(\partial c) \cap S_{j}^{-}=\emptyset$, for all $j \in E_{n-1}$. Thus, $\psi^{(t)}(\partial c)$ is contained in the closure of a connected component $c^{\prime}$ of

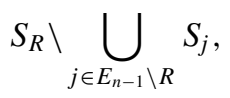

which is a cell of $\mathcal{A}(X \backslash\{n\})$ of dimension $\operatorname{dim} S_{R} \leq t+1$.

Theorem 5. There is an $(r-1)$-admissible embedding $\psi$ whose restriction to $(\mathcal{A}(X /$ $\{n\}))^{0}$ is compatible with $X$. It is unique, up to a homeomorphism $\mathbb{S}^{r-1} \rightarrow \mathbb{S}^{r-1}$ that fixes $\mathcal{A}(X \backslash\{n\})$ cellwise.

Proof. Let $\psi^{(t)}$ denote the restriction of $\psi$ to $(\mathcal{A}(X /\{n\}))^{(t-1)}$, for $t=1, \ldots, r-1$. We start with inductively proving the uniqueness of $\psi$. A 1 -admissible embedding $\psi^{(1)}$ that is compatible with $X$ is essentially unique, by Lemma 4 . For $t<r-1$, assume that $\psi^{(t)}$ is unique, up to a homeomorphism $\mathbb{S}^{r-1} \rightarrow \mathbb{S}^{r-1}$ that fixes $\mathcal{A}(X \backslash\{n\})$ cellwise. Let $c$ be a $t$-dimensional cell of $\mathcal{A}(X /\{n\})$, and let $R \subset E_{n-1}$ be maximal so that $\partial c \subset s_{R / n}$. If $\psi$ is $(r-1)$-admissible, then $\psi(c) \subset S_{R}$. Let $c^{\prime} \subset S_{R}$ be the cell of $\mathcal{A}(X \backslash\{n\})$ containing $\psi(c)$. Since $\mathcal{A}(X \backslash\{n\})$ is of full rank, the cell $c^{\prime}$ is uniquely determined by $\psi(\partial c)$. If $\operatorname{dim} c^{\prime}=\operatorname{dim} c$ then the $(r-1)$-admissibility of $\psi$ imposes $c^{\prime}=\psi(c)$. Otherwise, $\psi(c) \subset c^{\prime}$, being a tame ball of codimension one, is unique up to a homeomorphism $\bar{c}^{\prime} \rightarrow \bar{c}^{\prime}$ that fixes $\partial c^{\prime}$ pointwise, by the generalized Schönflies theorem. The homeomorphism can be extended to a homeomorphism $\mathbb{S}^{r-1} \rightarrow \mathbb{S}^{r-1}$ fixing all cells of $\mathcal{A}(X \backslash\{n\})$, by the cone construction. In conclusion, $\psi^{(t+1)}$ is essentially uniquely determined by $\psi^{(t)}$, which completes the proof of the uniqueness of $\psi$.

Secondly, we expose an iterative construction of $\psi$. A 1-admissible embedding $\psi^{(1)}$ that is compatible with $X$ exists, by Lemma 4 . For $t<r-1$, assume that $\psi^{(t)}$ is a 


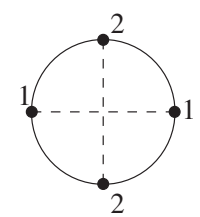

Fig. 4. Two linked 0 -spheres in $\mathbb{S}^{1}$.

$t$-admissible embedding, and let $c$ be a $t$-dimensional cell of $\mathcal{A}(X /\{n\})$. If there is a $t$ dimensional cell $c^{\prime}$ of $\mathcal{A}(X \backslash\{n\})$ such that $\partial c^{\prime}=\psi^{(t)}(\partial c)$ then we define $\psi^{(t+1)}(c)=c^{\prime}$. Otherwise, by the preceding lemma there is a $(t+1)$-dimensional cell $c^{\prime}$ of $\mathcal{A}(X \backslash\{n\})$ so that $\psi^{(t)}(\partial c) \subset \partial c^{\prime}$. Since $\psi^{(t)}(\partial c)$ is a tame union of tame cells of codimension one in $\partial c^{\prime}$, it follows from [14] that $\psi^{(t)}(\partial c)$ is a pseudosphere in $\partial c^{\prime}$. Therefore $\psi^{(t)}(\partial c)$ bounds a tame cell $c^{\prime \prime} \subset c^{\prime}$. We define $\psi^{(t+1)}(c)=c^{\prime \prime}$.

We show that we can do this construction so that $\psi^{(t+1)}$ is an embedding. Let $\Psi^{(t)}$ denote the image of $\psi^{(t)}$, and let $c^{\prime}$ be a $(t+1)$-dimensional cell of $\mathcal{A}(X \backslash\{n\})$. If $\psi^{(t)}$ is an embedding, then $\Psi^{(t)} \cap \partial c^{\prime}$ is a disjoint union of $t$-dimensional spheres. If $t>1$ then these spheres bound a system of disjoint $(t+1)$-dimensional cells in $c^{\prime}$. If $t=1$ then a priori the spheres might be linked, as depicted in Fig. 4. The thick dots indicate the images under $\psi$ of four zero-dimensional cells on a cycle of $\mathcal{A}(X /\{n\})$, whose cyclic order corresponds to the numbering. It is shown in [3] that this case does not occur. In conclusion, our construction of $\psi^{(t+1)}$ produces an embedding.

It remains to show that $\psi^{(t+1)}$ is $(t+1)$-admissible. Let $R \subset E_{n-1}$ with $\operatorname{dim} s_{R / n} \leq t$.

(1) Either $\psi^{(t+1)}\left(s_{R / n}\right)$ is equal to $S_{R}$ or it is composed of tame cells of codimension one in $S_{R}$. In the latter case, $\psi^{(t+1)}\left(s_{R / n}\right)$ is a pseudosphere (i.e., tame) by [14].

(2) Let $\psi^{(t+1)}\left(s_{R / n}\right) \neq S_{R}$, and let $\gamma \subset S_{R}$ be a cycle of $\mathcal{A}(X \backslash\{n\})$ that is not contained in $\psi^{(t+1)}\left(s_{R / n}\right)$. We choose a subset $T \subset E_{n-1}$ with $\gamma=S_{T}$ and $|T|=r-2$. The contraction $X / T$ is a hyperline sequence of rank 2 . Since $\psi^{(1)}$ is compatible with $X, \psi^{(t+1)}\left(s_{R / n}\right) \cap \gamma$ comprises exactly two points $x, y$, corresponding to the elements $n, \bar{n}$ in $X / T$.

There is some $x \in \mathbf{E}(X / T) \backslash\{n\}$ such that $X / T$ induces the cyclic order $(n, x, \bar{n}, \bar{x})$. Let $j=x^{*}$. Since $\psi^{(t)}$ is $t$-admissible, it follows from Lemma 2 that $\mathcal{A}(X \backslash\{n\})$ and $\psi^{(t+1)}\left(s_{R / n}\right)$ induce on $S_{R \cup\{j\}}$ an arrangement of oriented pseudospheres. If $\gamma$ does not meet both connected components of $S_{R} \backslash \psi^{(t+1)}\left(s_{R / n}\right)$, then the two points of $\gamma \cap S_{j}$ are contained in a single component of $S_{R \cup\{j\}} \backslash \psi^{(t+1)}$ $\left(s_{R / n}\right)$, which is impossible for arrangements of oriented pseudospheres. Hence, $\psi^{(t+1)}$ satisfies the second property in the definition of $(t+1)$-admissible embeddings.

(3) We observe that an open cell is contained in the positive side of an oriented pseudosphere if and only if some point in its boundary is contained in the positive side of the oriented pseudosphere. Thus, if $c$ is a $t$-dimensional cell of $\mathcal{A}(X /\{n\})$ and $c \subset s_{i}^{+}$, then $\partial c \cap s_{i}^{+} \neq \emptyset$. Since $\psi^{(t)}$ maps $s_{i}^{+}$into $S_{i}^{+}$, it follows that

$$
\psi^{(t)}(\partial c) \cap S_{i}^{+}=\psi^{(t+1)}(\partial c) \cap S_{i}^{+} \neq \emptyset,
$$

and therefore $\psi^{(t+1)}(c) \subset S_{i}^{+}$. Similarly, if $c \subset S_{i}^{-}$then $\psi^{(t+1)}(c) \subset S_{i}^{-}$.

Therefore $\psi^{(t+1)}$ is $(t+1)$-admissible, which finishes the proof of Theorem 5 . 
In the remainder of this section, we finish the proof of Theorem 4. Let $\psi: \mathbb{S}^{r-2} \rightarrow \mathbb{S}^{r-1}$ be an $(r-1)$-admissible embedding whose restriction to the 1-skeleton of $\mathcal{A}(X /\{n\})$ is compatible with $X$. By Theorem 5, $\psi$ exists. Let $S_{n}=\psi\left(\mathbb{S}^{r-2}\right)$ be the oriented pseudosphere defined by $\psi$, and set $\mathcal{A}=\left\{S_{1}, \ldots, S_{n}\right\}$. By Lemma $2, \mathcal{A}$ is an arrangement of oriented pseudospheres.

We show that $X(\mathcal{A})=X$, hence, that the hyperline sequences $(Y \mid Z) \in X$ coincide with those of $X(\mathcal{A})$. If $n \notin E(Y)$ then $(Y \mid Z) \in X(\mathcal{A})$, since $Z$ is a rank 2 contraction of $X$ and $\psi^{(1)}$ is 1-admissible and compatible with $X$. If $n \in E(Y)$, then let $R=E(Y) \backslash\{n\}$. Since $\psi$ is $(r-1)$-admissible, we have $S_{R} \subset S_{n}$, thus, $S_{R}$ corresponds to the cycle $s_{R / n} \subset \mathbb{S}^{r-2}$ of $\mathcal{A}(X /\{n\})$. Since $X(\mathcal{A}(X /\{n\}))=X /\{n\}$, the cyclic order of points on this cycle coincides with $Z$. If $i, j \in E(Z)$ so that $Z$ induces the cyclic order $(i, j, \bar{l}, \bar{J})$, then $X /\{i, j\}=Y$. We have $X(\mathcal{A} /\{i, j\})=X /\{i, j\}$, since the TRT holds in rank $r-2$ by the induction hypothesis. Therefore, $(Y \mid Z)=(X(\mathcal{A} /\{i, j\}) \mid Z) \in X(\mathcal{A})$.

It remains to prove the uniqueness of $\mathcal{A}$ stated in the TRT. Let $\left\{\tilde{S}_{1}, \ldots, \tilde{S}_{n}\right\}$ be an arrangement of oriented pseudospheres with $X\left(\left\{\tilde{S}_{1}, \ldots, \tilde{S}_{n}\right\}\right)=X$. By the induction hypothesis, we may use the uniqueness of $\mathcal{A}(X \backslash\{n\})$ and can assume that $\left\{\tilde{S}_{1}, \ldots, \tilde{S}_{n-1}\right\}=$ $\left\{S_{1}, \ldots, S_{n-1}\right\}$. Let $\tilde{S}_{n}$ be the image of a tame embedding $\tilde{\psi}: \mathbb{S}^{r-2} \rightarrow \mathbb{S}^{r-1}$. Then $\tilde{\psi}$ is $(r-1)$-admissible by Lemma 3 , and it is obvious that its restriction to the 1-skeleton of $\mathcal{A}(X /\{n\})$ is compatible with $X$. Thus, by Theorem $5, \tilde{\psi}$ coincides with $\psi$ up to a homeomorphism $\mathbb{S}^{r-1} \rightarrow \mathbb{S}^{r-1}$ that fixes $\left\{S_{1}, \ldots, S_{n-1}\right\}$ cellwise. Hence, $\mathcal{A}$ is equivalent to $\left\{\tilde{S}_{1}, \ldots, \tilde{S}_{n}\right\}$. This finishes the proof of the Topological Representation Theorem 4 .

\section{Wild Arrangements}

This section is an appendix to Section 5. We show here that one can read off an oriented matroid from an arrangement of oriented pseudospheres, even if one allows pseudospheres not to be tame. Let $S_{1}, \ldots, S_{n} \subset \mathbb{S}^{d}$ be embedded $(d-1)$-dimensional spheres with a choice of an orientation. We call the ordered multiset $\mathcal{A}=\left\{S_{1}, \ldots, S_{n}\right\}$ an arrangement of oriented topological spheres over $E_{n}$ if it satisfies Axioms (A1) and (A2), where the word "pseudosphere" is replaced by "embedded sphere of codimension one".

An embedded sphere $S \subset \mathbb{S}^{d}$ of codimension one is wild, if there is no homeomorphism $\mathbb{S}^{d} \rightarrow \mathbb{S}^{d}$ mapping $S$ to $\mathbb{S}^{d-1} \subset \mathbb{S}^{d}$. Since there are infinitely many wild spheres in $\mathbb{S}^{d}$ for all $d \geq 3$ (see [22]), we are no longer allowed to use the generalized Schönflies theorem. However, $\mathbb{S}^{d} \backslash S$ has exactly two connected components, even if $S$ is wild (see [23]).

Similarly to Section 4 , we say that two arrangements $\left\{S_{1}, \ldots, S_{n}\right\}$ and $\left\{\tilde{S}_{1}, \ldots, \tilde{S}_{n}\right\}$ of oriented topological spheres in $\mathbb{S}^{d}$ are equivalent if there is an orientation preserving homeomorphism $\varphi: \mathbb{S}^{d} \rightarrow \mathbb{S}^{d}$ with $\varphi\left(S_{i}\right)=\tilde{S}_{i}$ in the correct orientation, for $i=$ $1, \ldots, n$. For $R \subset E_{n}$, the definition of the contraction $\mathcal{A} / R$ and the deletion $\mathcal{A} \backslash R$ is identical to the corresponding definition for arrangements of oriented pseudospheres.

We wish to define an oriented matroid $X(\mathcal{A})$ given by hyperline sequences, associated to an arrangement $\mathcal{A}$ of oriented topological spheres. By Section 5, we know how to proceed if all spheres in $\mathcal{A}$ are tame. Both in the construction of $X(\mathcal{A})$ in Section 5 and in the proof that $X(\mathcal{A})$ is indeed an oriented matroid, we were using induction on the contractions of $\mathcal{A}$, based on contractions of ranks 1 and 2 . We verify that in the 
base cases and in the induction steps, we only used topological statements that are not based on tameness. Rank 1 is trivial. For rank 2, observe that any two different points $x, y \in \mathbb{S}^{1}$ can be separated by small intervals around $x$ and $y$. Thus, any embedded sphere $\mathbb{S}^{0}$ in $\mathbb{S}^{1}$ is tame, simply since $\mathbb{S}^{1}$ is a Hausdorff space. The only topological argument in the induction step was the use of the Jordan-Schönflies theorem, applied to a rank 3 contraction. The Jordan-Schönflies theorem does not assume tameness. Therefore, even if $\mathcal{A}$ is not equivalent to an arrangement of pseudospheres, any rank 2 contraction $\mathcal{A} / R$ actually is an arrangement of pseudospheres, and we can read off a hyperline sequence $X(\mathcal{A} / R)$ of rank 2 . As in Section 5, these contractions form an oriented matroid $X(\mathcal{A})$.

In conclusion, although arrangements of oriented topological spheres are very complicated from a topological point of view, their combinatorics is simple enough to read off ordinary oriented matroids. Nevertheless, there are "more" arrangements of oriented topological spheres than oriented matroids, in the sense that there are non-equivalent arrangements $\mathcal{A}_{1}$ and $\mathcal{A}_{2}$ of oriented topological spheres (for instance, a tame arrangement and a wild arrangement) with $X\left(\mathcal{A}_{1}\right)=X\left(\mathcal{A}_{2}\right)$. Hence, the uniqueness part of the TRT fails in the setting of arrangements of oriented topological spheres.

\section{Acknowledgments}

We thank Gerhard Burde, Klaus Johannson and Martin Scharlemann for their advice for topological references.

\section{References}

1. A. Björner, M. Las Vergnas, B. Sturmfels, N. White and G. Ziegler, Oriented Matroids, Cambridge University Press, Cambridge, 1993, second edition 1999.

2. J. Bokowski, Oriented matroids, in [11], pp. 555-602.

3. J. Bokowski, S. Mock and I. Streinu, On the Folkman-Lawrence topological representation theorem for oriented matroids of rank 3, European J. Combin., 22(5) (2001), 601-615.

4. M. Brown, A proof of the generalized Schoenflies theorem, Bull. Amer. Math. Soc., 66 (1960), 74-76.

5. M. Brown, Locally flat imbeddings of topological manifolds, Ann. of Math. (2), 77 (1962) 331-341.

6. J. Edmonds and A. Mandel, Topology of oriented matroids, Abstract 758-05-9, Notices Amer. Math. Soc., 25 (1978) A-410; also Ph.D. Thesis of A. Mandel, University of Waterloo, 1982.

7. J. Folkman and J. Lawrence, Oriented matroids, J. Combin. Theory Ser. B, 25 (1978), 199-236.

8. J.E. Goodman, Pseudoline arrangements, in [9], pp. 83-110.

9. J.E. Goodman and J. O'Rourke (eds.), Handbook of Combinatorial and Computational Geometry, CRC Press, Boca Raton, FL, 1997.

10. J.E. Goodman and R. Pollack, Semispaces of configurations, cell complexes of arrangements, J. Combin. Theory Ser. A, 37 (1984), 257-293

11. P.M. Gruber and J.M. Wills (eds.), Handbook of Convex Geometry, vols. A and B, North-Holland, Amsterdam, 1993.

12. L. Gutierrez Novoa, On n-ordered sets and order completeness, Pacific J. Math., 15 (1965), 1337-1345.

13. W. Hochstättler, Seitenflächenverbände orientierter Matroide, Ph.D. Thesis, Köln, 1992.

14. R.C. Kirby, The union of flat $(n-1)$-balls is flat in $R^{n}$, Bull. Amer. Math. Soc., 74 (1968), 614-617.

15. D.E. Knuth, Axioms and Hulls, Springer-Verlag, New York, 1992.

16. M. Las Vergnas, Matroïdes orientables, C.R. Acad. Sci. Paris Ser. A, 280 (1975), 61-64.

17. M. Las Vergnas, Bases in oriented matroids, J. Combin. Theory Ser. B, 25 (1978), 283-289. 
18. M. Las Vergnas, Extensions ponctuelles d'une géométrie combinatoire orientée, in Problèmes combinatoires et théorie des graphes (Colloq. Internat. CNRS, Univ. Orsay, Orsay, 1976), pp. 265-270, Colloq. Internat. CNRS 260, CNRS, Paris, 1978.

19. J. Lawrence, Oriented matroids and multiply ordered sets, Linear Algebra Appl., 48 (1982), 1-12.

20. E.E. Moise, Geometric Topology in Dimensions 2 and 3, Graduate Texts in Mathematics 47, SpringerVerlag, New York, 1977.

21. J. Richter-Gebert and G. Ziegler, Oriented matroids, in [9], pp. 111-132.

22. D. Rolfsen, Knots and Links, Mathematics Lecture Series 7, Publish or Perish, Houston, TX, 1976.

23. R. Stöcker and H. Zieschang, Algebraische Topologie. Eine Einführung, second edition, B. G. Teubner, Stuttgart, 1994.

24. E. Swartz, Representations of matroids, J. Amer. Math. Soc., 16 (2003), 427-442.

Received January 20, 2003, and in revised form September 27, 2004. Online publication March 22, 2005. 